\title{
Optimal Rational Approximations by the Modified Fourier Basis
}

\author{
Arnak V. Poghosyan $(1)$ and Tigran K. Bakaryan \\ Institute of Mathematics, Armenian National Academy of Sciences, 24/5 Marshal Baghramyan Ave., 0019 Yerevan, Armenia \\ Correspondence should be addressed to Arnak V. Poghosyan; arnak@instmath.sci.am
}

Received 18 October 2017; Accepted 20 February 2018; Published 1 April 2018

Academic Editor: Beong In Yun

Copyright (C) 2018 Arnak V. Poghosyan and Tigran K. Bakaryan. This is an open access article distributed under the Creative Commons Attribution License, which permits unrestricted use, distribution, and reproduction in any medium, provided the original work is properly cited.

\begin{abstract}
We consider convergence acceleration of the modified Fourier expansions by rational trigonometric corrections which lead to modified-trigonometric-rational approximations. The rational corrections contain some unknown parameters and determination of their optimal values for improved pointwise convergence is the main goal of this paper. The goal was accomplished by deriving the exact constants of the asymptotic errors of the approximations with further elimination of the corresponding main terms by appropriate selection of those parameters. Numerical experiments outline the convergence improvement of the optimal rational approximations compared to the expansions by the modified Fourier basis.
\end{abstract}

\section{Introduction}

The modified Fourier basis

$$
\begin{array}{r}
\mathscr{H}=\left\{\cos \pi n x: n \in \mathbb{Z}_{+}\right\} \cup\left\{\sin \pi\left(n-\frac{1}{2}\right) x: n \in \mathbb{N}\right\}, \\
x \in[-1,1]
\end{array}
$$

was originally proposed by Krein [1] and thoroughly investigated in a series of papers [2-10].

Let $M_{N}(f, x)$ be the truncated modified Fourier series

$$
\begin{aligned}
& M_{N}(f, x) \\
& \quad=\frac{1}{2} f_{0}^{c}+\sum_{n=1}^{N}\left[f_{n}^{c} \cos \pi n x+f_{n}^{s} \sin \pi\left(n-\frac{1}{2}\right) x\right],
\end{aligned}
$$

where

$$
\begin{aligned}
& f_{n}^{c}=\int_{-1}^{1} f(x) \cos \pi n x d x, \\
& f_{n}^{s}=\int_{-1}^{1} f(x) \sin \pi\left(n-\frac{1}{2}\right) x d x .
\end{aligned}
$$

Obviously, for even functions on $[-1,1]$, expansions by the modified Fourier basis coincide with the expansions by the classical Fourier basis

$$
\begin{aligned}
\mathscr{H}_{\text {class }}=\left\{\cos \pi n x: n \in \mathbb{Z}_{+}\right\} \cup\{\sin \pi n x: n & \in \mathbb{N}\}, \\
x & \in[-1,1] .
\end{aligned}
$$

Moreover, the modified Fourier basis can be derived from the other classical basis $\mathscr{H}^{*}$ on $[0,1]$

$$
\mathscr{H}^{*}=\left\{\cos \pi n x: n \in \mathbb{Z}_{+}\right\}, \quad x \in[0,1]
$$

by means of a change of variable.

The first results concerning the convergence of the expansions by the modified Fourier basis appeared in the works $[2,8-10]$. We present two theorems for further comparisons.

Theorem 1 (see [10]). Assume $f \in C^{2}[-1,1]$ and $f^{\prime \prime} \in$ $B V[-1,1]$. If $|x|<1$, then

$$
f(x)-M_{N}(f, x)=O\left(N^{-2}\right), \quad N \longrightarrow \infty .
$$

Otherwise,

$$
f( \pm 1)-M_{N}(f, \pm 1)=O\left(N^{-1}\right), \quad N \longrightarrow \infty .
$$


Under some additional requirements, the convergence rate is faster.

Theorem 2 (see $[2,10])$. Assume $f \in C^{2 q+2}(-1,1), f^{(2 q+2)} \in$ $B V[-1,1], q \geq 1$, and $f$ obeys the first $q$ derivative conditions:

$$
f^{(2 r+1)}( \pm 1)=0, \quad r=0, \ldots, q-1 .
$$

If $|x|<1$, then

$$
f(x)-M_{N}(f, x)=O\left(N^{-2 q-2}\right), \quad N \longrightarrow \infty .
$$

Otherwise,

$$
f( \pm 1)-M_{N}(f, \pm 1)=O\left(N^{-2 q-1}\right), \quad N \longrightarrow \infty .
$$

Overall, we see better convergence rates compared to the classical Fourier expansions [11]. This can be explained by faster decay of coefficients $f_{n}^{s}$ :

$$
f_{n}^{s}=O\left(n^{-2}\right), \quad n \longrightarrow \infty
$$

compared to the classical ones when $f$ is smooth enough but nonperiodic on $[-1,1]$. Estimate (11) can be explained by a nonperiodicity of the basis functions $\sin \pi(n-1 / 2) x$ on $[-1,1]$.

Convergence acceleration of the modified Fourier expansions by means of rational corrections was considered in [6]. Here, we continue those investigations. More specifically, consider a finite sequence of real numbers $\theta=\left\{\theta_{k}\right\}_{k=1}^{p}, p \geq 1$ and, by $\Delta_{n}^{k}(\theta, \widehat{f}), \widehat{f}=\left\{f_{n}\right\}$ denote the following generalized finite differences:

$$
\begin{aligned}
& \Delta_{n}^{0}(\theta, \widehat{f})=f_{n} \\
& \Delta_{n}^{k}(\theta, \widehat{f})=\Delta_{n}^{k-1}(\theta, \widehat{f})+\theta_{k} \Delta_{n-1}^{k-1}(\theta, \widehat{f}), \quad k \geq 1 .
\end{aligned}
$$

By $\Delta_{n}^{k}(\widehat{f})$, we denote the classical finite differences which correspond to generalized differences $\Delta_{n}^{k}(\theta, \widehat{f})$ with $\theta \equiv 1$. It is easy to verify that

$$
\Delta_{n}^{k}(\widehat{f})=\sum_{\ell=0}^{k}\left(\begin{array}{l}
k \\
\ell
\end{array}\right) f_{n-\ell} .
$$

Let

$$
\begin{aligned}
R_{N}(f, x) & =f(x)-M_{N}(f, x) \\
& =R_{N}^{c}(f, x)+R_{N}^{s}(f, x),
\end{aligned}
$$

where

$$
\begin{aligned}
& R_{N}^{c}(f, x)=\sum_{n=N+1}^{\infty} f_{n}^{c} \cos \pi n x, \\
& R_{N}^{s}(f, x)=\sum_{n=N+1}^{\infty} f_{n}^{s} \sin \pi\left(n-\frac{1}{2}\right) x .
\end{aligned}
$$

Consider two sequences of real numbers $\theta^{c}=\left\{\theta_{k}^{c}\right\}_{k=1}^{p}$ and $\theta^{s}=\left\{\theta_{k}^{s}\right\}_{k=1}^{p}$. Let $\widehat{f}^{s}=\left\{f_{n}^{s}\right\}_{n=1}^{\infty}$ and $\widehat{f}^{c}=\left\{f_{n}^{c}\right\}_{n=0}^{\infty}$. Let $\mu_{j}(k, \theta)$ be defined by the following identities:

$$
\prod_{j=1}^{k}\left(1+\theta_{j} x\right)=\sum_{j=0}^{k} \mu_{j}(k, \theta) x^{j}, \quad k=1, \ldots, p .
$$

By means of sequential Abel transformations (see details in [6]), we derive the following expansions of errors (15):

$$
\begin{aligned}
& R_{N}^{c}(f, x)=-\sum_{k=1}^{p} \frac{\theta_{k}^{c} \Delta_{N}^{k-1}\left(\theta^{c}, \widehat{f}^{c}\right)}{\prod_{r=1}^{k}\left(1+2 \theta_{r}^{c} \cos \pi x+\left(\theta_{r}^{c}\right)^{2}\right)} \\
& \cdot \sum_{j=0}^{k} \mu_{j}\left(k, \theta^{c}\right) \cos \pi(N+1-j) x+R_{N, p}^{c}\left(f, \theta^{c}, x\right), \\
& R_{N}^{s}(f, x)=-\sum_{k=1}^{p} \frac{\theta_{k}^{s} \Delta_{N}^{k-1}\left(\theta^{s}, \widehat{f}^{s}\right)}{\prod_{r=1}^{k}\left(1+2 \theta_{r}^{s} \cos \pi x+\left(\theta_{r}^{s}\right)^{2}\right)} \\
& \quad \cdot \sum_{j=0}^{k} \mu_{j}\left(k, \theta^{s}\right) \sin \pi\left(N+\frac{1}{2}-j\right) x \\
& +R_{N, p}^{s}\left(f, \theta^{s}, x\right),
\end{aligned}
$$

where

$$
\begin{aligned}
R_{N, p}^{c}(f, \theta, x) & \frac{1}{2 \prod_{k=1}^{p}\left(1+\theta_{k} e^{i \pi x}\right)} \sum_{n=N+1}^{\infty} \Delta_{n}^{p}\left(\theta, \widehat{f}^{c}\right) e^{i \pi n x} \\
& +\frac{1}{2 \prod_{k=1}^{p}\left(1+\theta_{k} e^{-i \pi x}\right)} \sum_{n=N+1}^{\infty} \Delta_{n}^{p}\left(\theta, \widehat{f}^{c}\right) e^{-i \pi n x}, \\
R_{N, p}^{s}(f, \theta, x) & \frac{e^{-i \pi x / 2}}{2 i \prod_{k=1}^{p}\left(1+\theta_{k} e^{i \pi x}\right)} \sum_{n=N+1}^{\infty} \Delta_{n}^{p}\left(\theta, \widehat{f}^{s}\right) e^{i \pi n x} \\
& -\frac{e^{i \pi x / 2}}{2 i \prod_{k=1}^{p}\left(1+\theta_{k} e^{-i \pi x}\right)} \sum_{n=N+1}^{\infty} \Delta_{n}^{p}\left(\theta, \widehat{f}^{s}\right) e^{-i \pi n x} .
\end{aligned}
$$

These expansions lead to the following modified-trigonometric-rational (MTR-) approximations:

$$
\begin{aligned}
& M_{N, p}\left(f, \theta^{c}, \theta^{s}, x\right)=M_{N}(f, x) \\
& -\sum_{k=1}^{p} \frac{\theta_{k}^{c} \Delta_{N}^{k-1}\left(\theta^{c}, \widehat{f}^{c}\right)}{\prod_{r=1}^{k}\left(1+2 \theta_{r}^{c} \cos \pi x+\left(\theta_{r}^{c}\right)^{2}\right)} \sum_{j=0}^{k} \mu_{j}\left(k, \theta^{c}\right) \\
& \cdot \cos \pi(N+1-j) x \\
& -\sum_{k=1}^{p} \frac{\theta_{k}^{s} \Delta_{N}^{k-1}\left(\theta^{s}, \widehat{f}^{s}\right)}{\prod_{r=1}^{k}\left(1+2 \theta_{r}^{s} \cos \pi x+\left(\theta_{r}^{s}\right)^{2}\right)} \sum_{j=0}^{k} \mu_{j}\left(k, \theta^{s}\right) \\
& \cdot \sin \pi\left(N+\frac{1}{2}-j\right) x,
\end{aligned}
$$


with the error

$$
\begin{aligned}
R_{N, p}\left(f, \theta^{c}, \theta^{s}, x\right) & =f(x)-M_{N, p}\left(f, \theta^{c}, \theta^{s}, x\right) \\
& =R_{N, p}^{c}\left(f, \theta^{c}, x\right)+R_{N, p}^{s}\left(f, \theta^{s}, x\right) .
\end{aligned}
$$

A crucial step for realization of the rational approximations is determination of parameters $\theta^{c}$ and $\theta^{s}$. Different approaches are known for solution of this problem (see [1219]). In general, appropriate determination of these parameters leads to rational approximations with improved accuracy compared to the classical ones in case of smooth $f$. However, the rational approximations are essentially nonlinear in the sense that

$$
\begin{aligned}
& M_{N, p}\left(f+g, \theta^{c}, \theta^{s}, x\right) \\
& \quad \neq M_{N, p}\left(f, \theta^{c}, \theta^{s}, x\right)+M_{N, p}\left(g, \theta^{c}, \theta^{s}, x\right)
\end{aligned}
$$

as for each approximation we need to determine its own $\theta^{c}$ and $\theta^{s}$ vectors.

In [6], those parameters were determined from the following systems of equations:

$$
\begin{aligned}
& \Delta_{n}^{p}\left(\theta^{c}, \widehat{f}^{c}\right)=0, \quad n=N, N-1, \ldots, N-p+1, \\
& \Delta_{n}^{p}\left(\theta^{s}, \widehat{f}^{s}\right)=0, \quad n=N, N-1, \ldots, N-p+1,
\end{aligned}
$$

which led to the Fourier-Pade type approximations [12] with better convergence for smooth functions (see [6]) compared to the expansions by the modified Fourier basis. It is rather complex approach as parameters $\theta^{c}$ and $\theta^{s}$ depend on $N$ and systems (23) must be solved for each $N$.

In this paper, assuming that $f$ is smooth on $[-1,1]$, we consider simpler alternative approach, where $\theta^{s}$ and $\theta^{c}$ are determined as follows $[14,16,19]$ :

$$
\begin{aligned}
& \theta_{k}^{c}=1-\frac{\tau_{k}^{c}}{N}, \\
& \theta_{k}^{s}=1-\frac{\tau_{k}^{s}}{N},
\end{aligned}
$$

$$
\tau_{k}^{c} \neq 0, \quad \tau_{k}^{s} \neq 0, k=1, \ldots, p,
$$

with $\tau^{c}=\left\{\tau_{1}^{c}, \ldots, \tau_{p}^{c}\right\}$ and $\tau^{s}=\left\{\tau_{1}^{s}, \ldots, \tau_{p}^{s}\right\}$ independent of $N$. Actually, in this approach, we take into consideration only the first two terms of the asymptotic expansions of $\theta_{k}=$ $\theta_{k}(N)$ in terms of $1 / N$. Although parameters $\theta^{c}$ and $\theta^{s}$ in (24) depend on $N$, we need only to determine $\tau^{c}$ and $\tau^{s}$ which are independent of $N$. Hence, this approach is less complex than the modified Fourier-Pade approximations.

The main results of this paper are exact constants of the main terms of asymptotic errors and optimal parameters for improved pointwise convergence of rational approximations. First, we derive the exact estimates for the main terms of asymptotic errors without specifying parameters $\tau^{c}$ and $\tau^{s}$. Second, we determine the optimal values of parameters $\tau^{c}$ and $\tau^{s}$ which vanish the main terms and lead to approximations with substantially better pointwise convergence rates. We found that optimal values of parameters $\tau_{k}^{c}$ and $\tau_{k}^{s}$, $k=1, \ldots, p$, are the roots of some polynomials depending on $p$ and $q$, where $q$ indicates the number of zero derivatives in (8). Moreover, the choice of optimal parameters depends on the parity of $p$ and also on the location of $x$, whether $|x|<1$ or $x= \pm 1$.

For example, when $p$ is odd and $|x|<1$, the roots of the generalized Laguerre polynomial $L_{p}^{(2 q+1)}(x)$ (see Appendix A) could be used for $\tau^{c}$ and $\tau^{\mathcal{s}}$. In this case, the convergence rate of the MTR-approximations is $O\left(N^{-2 q-p-[(p+1) / 2]-2}\right)$ as $N \rightarrow$ $\infty$. It means better convergence compared to the expansions by the modified Fourier basis with improvement by factor $O\left(N^{p+[(p+1) / 2]}\right)$. When $p$ is odd and $x= \pm 1$, the roots of the generalized Laguerre polynomial $L_{p}^{(2 q)}(x)$ could be used. In this case, the convergence rate is $O\left(N^{-2 q-[(p+1) / 2]-1}\right)$ with the improvement by factor $O\left(N^{[(p+1) / 2]}\right)$. The problem that we encountered is impossibility to get simultaneous optimality on $|x|<1$ and at $x= \pm 1$. One must decide whether to use parameters that provide the minimal error on $[-1,1]$, but with worse accuracy on $|x|<1$, or work with optimal parameters on $|x|<1$ with worse accuracy at $x= \pm 1$. We expose similar observations for even values of $p$.

It is important that the values of parameters $\tau^{c}$ and $\tau^{s}$ depend only on $p$ and $q$ and are independent of $f$. It means that if functions $f, g$, and $f+g$ have enough smoothness and obey the same derivative conditions, the optimal approach leads to linear rational approximations in the sense that

$$
\begin{aligned}
& M_{N, p}\left(f+g, \theta^{c}, \theta^{s}, x\right) \\
& \quad=M_{N, p}\left(f, \theta^{c}, \theta^{s}, x\right)+M_{N, p}\left(g, \theta^{c}, \theta^{s}, x\right)
\end{aligned}
$$

with the same parameters $\theta^{c}$ and $\theta^{s}$ for all included functions.

The paper is organized as follows. Section 2 presents some preliminary lemmas. Section 3 explores the pointwise convergence of the MTR-approximations away from the endpoints when $|x|<1$. Section 4 considers the pointwise convergence of the MTR-approximations when $x= \pm 1$. In these sections, we show also the results of numerical experiments which confirm and explain the theoretical findings. Section 5 presents some concluding remarks. Appendix A recalls some results concerning the Laguerre polynomials and Appendix B proves some combinatorial identities that we used in the proofs of lemmas and theorems.

\section{Preliminaries}

Throughout the paper, we assume that parameters $\theta_{k}, k=$ $1, \ldots, p$ are defined by (see (24))

$$
\theta_{k}=1-\frac{\tau_{k}}{N}, \quad \tau_{k} \neq 0, k=1, \ldots, p
$$

Let $\tau=\left\{\tau_{1}, \ldots, \tau_{p}\right\}$ and let coefficients $\gamma_{k}(\tau)$ be defined by the following identity:

$$
\prod_{k=1}^{p}\left(1+\tau_{k} x\right)=\sum_{k=0}^{p} \gamma_{k}(\tau) x^{k} .
$$


For $f \in C^{2 q+1}[-1,1], q \geq 0$, we put

$$
\begin{array}{r}
A_{2 k+1}(f)=\left(f^{(2 k+1)}(1)-f^{(2 k+1)}(-1)\right)(-1)^{k}, \\
k=0, \ldots, q, \\
B_{2 k+1}(f)=\left(f^{(2 k+1)}(1)+f^{(2 k+1)}(-1)\right)(-1)^{k}, \\
k=0, \ldots, q .
\end{array}
$$

The following estimates can be simply obtained by means of integration by parts of the integrals in (3).

Lemma 3. Assume $f \in C^{2 m+1}[-1,1], m \geq 0$, and $f^{(2 m+1)} \in$ $B V[-1,1]$. Then, the following asymptotic expansions are valid:

$$
\begin{aligned}
& f_{n}^{c}=(-1)^{n} \sum_{k=0}^{m} \frac{A_{2 k+1}(f)}{(\pi n)^{2 k+2}}+o\left(n^{-2 m-2}\right), \quad n \longrightarrow \infty, \\
& f_{n}^{s}=(-1)^{n+1} \sum_{k=0}^{m} \frac{B_{2 k+1}(f)}{(\pi(n-1 / 2))^{2 k+2}}+o\left(n^{-2 m-2}\right),
\end{aligned}
$$

$$
n \longrightarrow \infty \text {. }
$$

Lemma 4. Assume $f \in C^{2 m+2}[-1,1], m \geq 0$, and $f^{(2 m+2)} \in$ $B V[-1,1]$. Then, the following asymptotic expansions are valid:

$$
\begin{aligned}
f_{n}^{c} & =(-1)^{n} \sum_{k=0}^{m} \frac{A_{2 k+1}(f)}{(\pi n)^{2 k+2}}+o\left(n^{-2 m-3}\right), \quad n \longrightarrow \infty, \\
f_{n}^{s}=(-1)^{n+1} \sum_{k=0}^{m} \frac{B_{2 k+1}(f)}{(\pi(n-1 / 2))^{2 k+2}}+o\left(n^{-2 m-3}\right), & \\
n & \longrightarrow \infty .
\end{aligned}
$$

Next lemma unveils the asymptotic expansions of $\Delta^{w}\left(\widehat{\Delta}^{p}\left(\theta, \widehat{f}^{c}\right)\right)$ and $\Delta^{w}\left(\widehat{\Delta}^{p}\left(\theta, \widehat{f}^{s}\right)\right)$, where

$$
\widehat{\Delta}^{p}(\theta, \widehat{f})=\left\{\Delta_{n}^{p}(\theta, \widehat{f})\right\}_{n}
$$

Lemma 5. Assume $f \in C^{2 q+p+r+1}[-1,1], f^{(2 q+p+r+1)} \in$ $B V[-1,1], q \geq 0, r \geq 0, p \geq 1$, and

$$
f^{(2 k+1)}( \pm 1)=0, \quad k=0, \ldots, q-1
$$

Let $\theta_{k}, k=1, \ldots, p$, be defined by (26). Then, the following estimates hold for $n>N$ as $N \rightarrow \infty$ :

$$
\begin{aligned}
& \Delta_{n}^{w}\left(\widehat{\Delta}^{p}\left(\theta, \widehat{f}^{c}\right)\right)=(-1)^{n} \sum_{k=0}^{p} \frac{\gamma_{k}(\tau)}{N^{k}} \\
& \cdot \sum_{t=2 q+w}^{2 q+r} \frac{(t+p-k+1) !}{n^{t+p-k+2}} \sum_{s=q}^{[(t-w) / 2]} \frac{A_{2 s+1}(f)}{\pi^{2 s+2}(2 s+1) !} \\
& \cdot \beta_{k, s, t}(w)+\frac{o\left(N^{-p}\right)}{n^{2 q+r+2}},
\end{aligned}
$$

$$
\begin{aligned}
& \Delta_{n}^{w}\left(\widehat{\Delta}^{p}\left(\theta, \widehat{f}^{s}\right)\right)=(-1)^{n+1} \sum_{k=0}^{p} \frac{\gamma_{k}(\tau)}{N^{k}} \\
& \cdot \sum_{t=2 q+w}^{2 q+r} \frac{(t+p-k+1) !}{n^{t+p-k+2}} \sum_{s=q}^{[(t-w) / 2]} \frac{B_{2 s+1}(f)}{\pi^{2 s+2}(2 s+1) !} \\
& \cdot \widetilde{\beta}_{k, s, t}(w)+\frac{o\left(N^{-p}\right)}{n^{2 q+r+2}},
\end{aligned}
$$

where

$$
\begin{aligned}
& \beta_{k, s, t}(w)=\sum_{\ell=w}^{t-2 s} k^{t-2 s-\ell} \frac{\alpha_{w+p-k, \ell+p-k}}{(t-2 s-\ell) !(p-k+\ell) !}, \\
& \widetilde{\beta}_{k, s, t}(w) \\
& =\sum_{\ell=w}^{t-2 s}\left(k+\frac{1}{2}\right)^{t-2 s-\ell} \frac{\alpha_{w+p-k, \ell+p-k}}{(t-2 s-\ell) !(p-k+\ell) !},
\end{aligned}
$$

with

$$
\alpha_{k, j}=\sum_{s=0}^{k}\left(\begin{array}{l}
k \\
s
\end{array}\right)(-1)^{s} s^{j}, \quad j \geq 0 .
$$

Proof. In view of (12) and (26), we have

$$
\Delta_{n}^{p}\left(\theta, \widehat{f}^{c}\right)=\sum_{k=0}^{p} \frac{(-1)^{k} \gamma_{k}(\tau)}{N^{k}} \Delta_{n-k}^{p-k}\left(\widehat{f}^{c}\right) .
$$

Taking into account the fact that $\Delta_{n}^{w}\left(\widehat{\Delta}^{p-k}\left(\widehat{f}^{c}\right)\right)=\Delta_{n}^{w+p-k}\left(\widehat{f}^{c}\right)$ and using (13), we get

$$
\begin{aligned}
\Delta_{n}^{w} & \left(\widehat{\Delta}^{p}\left(\theta, \hat{f}^{c}\right)\right) \\
& =\sum_{k=0}^{p} \frac{(-1)^{k} \gamma_{k}(\tau)}{N^{k}} \sum_{j=0}^{w+p-k}\left(\begin{array}{c}
w+p-k \\
j
\end{array}\right) f_{n-(k+j)}^{c} .
\end{aligned}
$$

Application of Lemma 3, when $p+r$ is even, and Lemma 4, when $p+r$ is odd, leads to the following asymptotic expansion $(h=p-k+r)$ :

$$
\begin{aligned}
\Delta_{n}^{w}\left(\widehat{\Delta}^{p}\left(\theta, \widehat{f}^{c}\right)\right)= & (-1)^{n} \sum_{k=0}^{p} \frac{\gamma_{k}(\tau)}{N^{k}} \\
& \cdot \sum_{j=0}^{w+p-k}(-1)^{j}\left(\begin{array}{c}
w+p-k \\
j
\end{array}\right) \\
& \cdot \sum_{s=q}^{q+[h / 2]} \frac{A_{2 s+1}(f)}{(\pi n)^{2 s+2}} \\
& \cdot \frac{1}{(1-(j+k) / n)^{2 s+2}}+\frac{o\left(N^{-p}\right)}{n^{2 q+r+2}} .
\end{aligned}
$$


Then,

$$
\begin{aligned}
\Delta_{n}^{w}\left(\widehat{\Delta}^{p}\left(\theta, \hat{f}^{c}\right)\right)= & (-1)^{n} \sum_{k=0}^{p} \frac{\gamma_{k}(\tau)}{N^{k}} \\
& \cdot \sum_{j=0}^{w+p-k}(-1)^{j}\left(\begin{array}{c}
w+p-k \\
j
\end{array}\right) \\
& \cdot \sum_{s=q}^{q+[h / 2]} \frac{A_{2 s+1}(f)}{(\pi n)^{2 s+2}} \sum_{t=2 s+1}^{\infty}\left(\begin{array}{c}
t \\
2 s+1
\end{array}\right) \\
& \cdot \frac{(k+j)^{t-2 s-1}}{n^{t-2 s-1}}+\frac{o\left(N^{-p}\right)}{n^{2 q+r+2}} .
\end{aligned}
$$

Finally,

$$
\begin{aligned}
\Delta_{n}^{w}\left(\widehat{\Delta}^{p}\left(\theta, \widehat{f}^{c}\right)\right)= & (-1)^{n} \sum_{k=0}^{p} \frac{\gamma_{k}(\tau)}{N^{k}} \sum_{t=2 q}^{2 q+h} \frac{1}{n^{t+2}} \\
& \cdot \sum_{s=q}^{[t / 2]} \frac{A_{2 s+1}(f)}{\pi^{2 s+2}} \\
& \cdot\left(\begin{array}{c}
t+1 \\
t-2 s
\end{array}\right) \sum_{\ell=0}^{t-2 s}\left(\begin{array}{c}
t-2 s \\
\ell
\end{array}\right) \\
& \cdot k^{t-2 s-\ell} \alpha_{w+p-k, \ell}+\frac{o\left(N^{-p}\right)}{n^{2 q+r+2}} .
\end{aligned}
$$

It remains to notice that $\alpha_{w+p-k, \ell}=0$ for $0 \leq \ell<w+p-k$ (see [20]). Hence, $t-2 s \geq w+p-k$, and $s \leq[(t-w) / 2]$.

\section{Pointwise Convergence Away from the Endpoints}

In this section, we explore the pointwise convergence of the MTR-approximations away from the endpoints. Next theorem reveals the asymptotic behavior of the MTRapproximations for $|x|<1$ without specifying the selection of parameters $\tau^{c}$ and $\tau^{s}$.

Theorem 6. Assume $f \in C^{2 q+p+2}[-1,1], f^{(2 q+p+2)} \in B V[-1$, 1], $q \geq 0, p \geq 1$, and

$$
f^{(2 k+1)}( \pm 1)=0, \quad k=0, \ldots, q-1 .
$$

Let $\theta_{k}, k=1, \ldots, p$, be defined by (26). Then, the following estimates hold for $|x|<1$ as $N \rightarrow \infty$ :

$$
\begin{aligned}
& R_{N, p}^{c}(f, \theta, x)=A_{2 q+1}(f) \\
& \cdot \frac{(-1)^{N+p+1}}{N^{2 q+p+2} 2^{p+1} \pi^{2 q+2}(2 q+1) !} \\
& \quad \cdot \frac{\cos (\pi x / 2)(2 N-p+1)}{\cos ^{p+1}(\pi x / 2)} h_{p, 2 q+1}(\tau) \\
& \quad+o\left(N^{-2 q-p-2}\right),
\end{aligned}
$$

$$
\begin{aligned}
R_{N, p}^{s}(f, \theta, x)=B_{2 q+1}(f) \\
\cdot \frac{(-1)^{N+p}}{N^{2 q+p+2} 2^{p+1} \pi^{2 q+2}(2 q+1) !} \\
\quad \cdot \frac{\sin (\pi x / 2)(2 N-p)}{\cos ^{p+1}(\pi x / 2)} h_{p, 2 q+1}(\tau)+o\left(N^{-2 q-p-2}\right),
\end{aligned}
$$

where

$$
h_{p, m}(\tau)=\sum_{k=0}^{p}(-1)^{k} \gamma_{k}(\tau)(m+p-k) !
$$

Proof. We prove only estimate (44). Estimate (45) can be handled similarly.

Taking into account the fact that $\theta_{k} \rightarrow 1$ as $N \rightarrow \infty$, we estimate only the sums on the right-hand side of (18). By the Abel transformation, we get

$$
\begin{aligned}
\sum_{n=N+1}^{\infty} \Delta_{n}^{p}\left(\theta, \widehat{f}^{c}\right) e^{ \pm i \pi n x} \\
=-\frac{e^{ \pm i \pi(N+1) x}}{\left(1+e^{ \pm i \pi x}\right)} \Delta_{N}^{p}\left(\theta, \widehat{f}^{c}\right) \\
\quad-\frac{e^{ \pm i \pi(N+1) x}}{\left(1+e^{ \pm i \pi x}\right)^{2}} \Delta_{N}^{1}\left(\widehat{\Delta}^{p}\left(\theta, \widehat{f}^{c}\right)\right) \\
+\frac{1}{\left(1+e^{ \pm i \pi x}\right)^{2}} \sum_{n=N+1}^{\infty} \Delta_{n}^{2}\left(\widehat{\Delta}^{p}\left(\theta, \widehat{f}^{c}\right)\right) e^{ \pm i \pi n x} .
\end{aligned}
$$

Lemma 5 estimates sequences $\Delta_{N}^{p}\left(\theta, \widehat{f}^{c}\right), \Delta_{N}^{1}\left(\widehat{\Delta}^{p}\left(\theta, \hat{f}^{c}\right)\right)$, and $\Delta_{n}^{2}\left(\widehat{\Delta}^{p}\left(\theta, \widehat{f}^{c}\right)\right)$ as $N \rightarrow \infty$ and $n \geq N+1$. It shows that, for $r=1$ and $w=2$, we have

$$
\Delta_{n}^{2}\left(\widehat{\Delta}^{p}\left(\theta, \hat{f}^{c}\right)\right)=\frac{o\left(N^{-p}\right)}{n^{2 q+3}},
$$

and the third term in the right-hand side of (47) is $o\left(N^{-p-2 q-2}\right)$. Then, with $r=1$ and $w=1$, we have

$$
\Delta_{N}^{1}\left(\widehat{\Delta}^{p}\left(\theta, \widehat{f}^{c}\right)\right)=O\left(N^{-p-2 q-3}\right),
$$

and the second term is $O\left(N^{-p-2 q-3}\right)$. Finally, using the exact estimate for $\Delta_{N}^{p}\left(\theta, \widehat{f}^{c}\right)$, we derive

$$
\begin{aligned}
\Delta_{N}^{p}\left(\theta, \hat{f}^{c}\right)= & A_{2 q+1}(f) \frac{(-1)^{N}}{N^{p+2 q+2} \pi^{2 q+2}} \\
& \cdot \sum_{k=0}^{p} \gamma_{k}(\tau)\left(\begin{array}{c}
2 q+p-k+1 \\
2 q+1
\end{array}\right) \alpha_{p-k, p-k} \\
& +O\left(N^{-2 q-p-3}\right),
\end{aligned}
$$

which completes the proof as

$$
\alpha_{p-k, p-k}=(-1)^{p-k}(p-k) !
$$




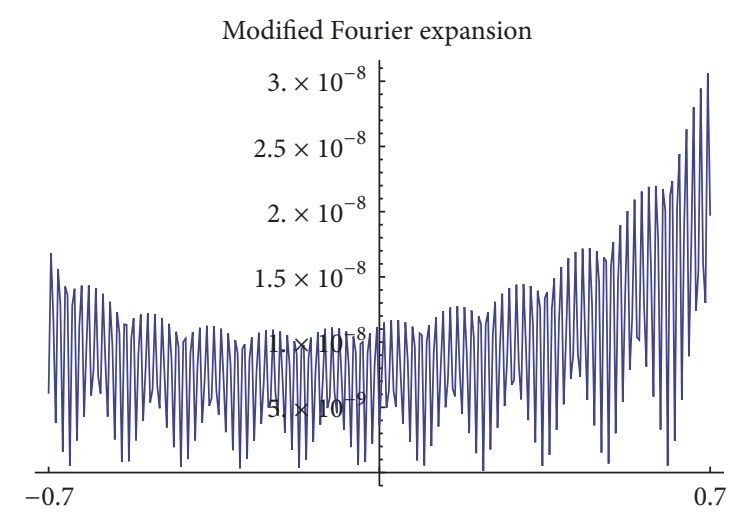

FIgURE 1: The graph of $\left|R_{N}(f, x)\right|$ on $[-0.7,0.7]$ for $N=64$ while approximating (53) by the modified Fourier expansion (2).

Note that Theorem 6 is valid also for $p=0$ which corresponds to the modified Fourier expansions (compare with Theorems 1 and 2). In that case, the exact constants of the main terms in (44) and (45) coincide with similar estimate in [3] (Theorem 2.22, page 29).

Theorem 6 shows that

$$
\begin{aligned}
R_{N, p}\left(f, \theta^{c}, \theta^{s}, x\right) & =R_{N, p}^{c}\left(f, \theta^{c}, x\right)+R_{N, p}^{s}\left(f, \theta^{s}, x\right) \\
& =O\left(N^{-2 q-p-2}\right)
\end{aligned}
$$

if parameters $\theta^{c}$ and $\theta^{s}$ are defined by (24). We see improvement in convergence rate by factor $O\left(N^{p}\right)$ and this result is obtained without specifying parameters $\tau^{c}$ and $\tau^{s}$.

Let us compare the modified Fourier expansions and MTR-approximations for a specific smooth function. Consider the following one:

$$
f(x)=\left(1-x^{2}\right)^{2} \sin (x-1)
$$

for which

$$
\begin{aligned}
f(1) & =f(-1)=0, \\
f^{\prime}(1) & =f^{\prime}(-1)=0, \\
f^{\prime \prime}(1) & =0, \\
f^{\prime \prime}(-1) & =-8 \sin (2), \\
f^{\prime \prime \prime}(1) & =24, \\
f^{\prime \prime \prime}(-1) & =24 \cos (2)+24 \sin (2) .
\end{aligned}
$$

Hence, this function obeys first $q=1$ derivative conditions (8).

Figures 1 and 2 show the behaviors of $\left|R_{N}\right|(p=0)$ and $\left|R_{N, p}\right|(p=1,2,3,4)$, respectively, on interval [-0.7,0.7] for $N=64$ while approximating (53). We used $\tau_{k}^{c}=\tau_{k}^{s}=k$, $k=1, \ldots, p$ in the rational approximations.

According to the results of Theorem 6, the bigger the value of $p$ is, the higher the accuracy of the corresponding approximations is. We observe it empirically. We see that $\max _{[-0.7,0.7]}\left|R_{N, p}\right|$ is $3 \cdot 10^{-8}$ for $p=0$, is $1.6 \cdot 10^{-9}$ for $p=1$, is $9 \cdot 10^{-11}$ for $p=2$, is $6.6 \cdot 10^{-12}$ for $p=3$, and is $5.7 \cdot 10^{-13}$ for $p=4$.

Can we improve the accuracy of the rational approximations by appropriate selection of parameters $\tau^{s}$ and $\tau^{c}$ ? Further, in this section, we give positive answer to this question and show how the optimal values can be chosen.

Estimates of Theorem 6 show that improvement can be achieved if parameters are chosen such that $\tau^{\mathcal{s}}=\tau^{c}=\tau$ and

$$
h_{p, 2 q+1}(\tau)=0 .
$$

By looking into the definition of $h_{p, 2 q+1}(\tau)$, we observe that condition (55) can be achieved, for example, if

$$
\gamma_{k}(\tau)=\left(\begin{array}{l}
p \\
k
\end{array}\right) \frac{(2 q+1+p) !}{(2 q+1+p-k) !} Q_{r}(k),
$$

where $Q_{r}(k)$ is a polynomial of order $r \leq p-1$

$$
Q_{r}(k)=\sum_{j=0}^{r} c_{j} k^{j}, \quad c_{0}=1,
$$

with unknown coefficients $c_{j}, j=1, \ldots, r$. Then, condition (55) follows from the well-known identity

$$
\sum_{k=0}^{p}(-1)^{k}\left(\begin{array}{l}
p \\
k
\end{array}\right) k^{j}=0, \quad j<p .
$$

Further, we determine the values of $c_{j}, j=1, \ldots, r$, for improved convergence of the rational approximations.

Next result is an immediate consequence of those observations and estimates of Theorem 6.

Theorem 7. Let $f \in C^{2 q+p+2}[-1,1], f^{(2 q+p+2)} \in B V[-1,1]$, $q \geq 0, p \geq 1$, and

$$
f^{(2 k+1)}( \pm 1)=0, \quad k=0, \ldots, q-1 .
$$

Assume the polynomial

$$
\sum_{k=0}^{p}\left(\begin{array}{l}
p \\
k
\end{array}\right) \frac{Q_{r}(p-k)}{(2 q+1+k) !}(-1)^{k} x^{k}
$$

has only real-valued and nonzero roots $x=z_{k}, k=1, \ldots, p$, and let

$$
\theta_{k}^{c}=\theta_{k}^{s}=1-\frac{z_{k}}{N}, \quad k=1, \ldots, p .
$$

Then, the following estimate holds for $|x|<1$ :

$$
R_{N, p}\left(f, \theta^{c}, \theta^{s}, x\right)=o\left(N^{-2 q-p-2}\right), \quad N \longrightarrow \infty .
$$

Theorem 7 is valid only if, for given $p$ and $q$, polynomial (60) has only real-valued and nonzero roots. Further, we clarify this statement by showing those cases when it is true.

By imposing extra smoothness on the underlying functions, we derive more precise estimate of (62). $\left.\left.\widehat{f}^{s}\right)\right)$.

First, we need estimates for $\Delta_{N}^{w}\left(\widehat{\Delta}^{p}\left(\theta, \widehat{f}^{c}\right)\right)$ and $\Delta_{N}^{w}\left(\widehat{\Delta}^{p}(\theta\right.$, 

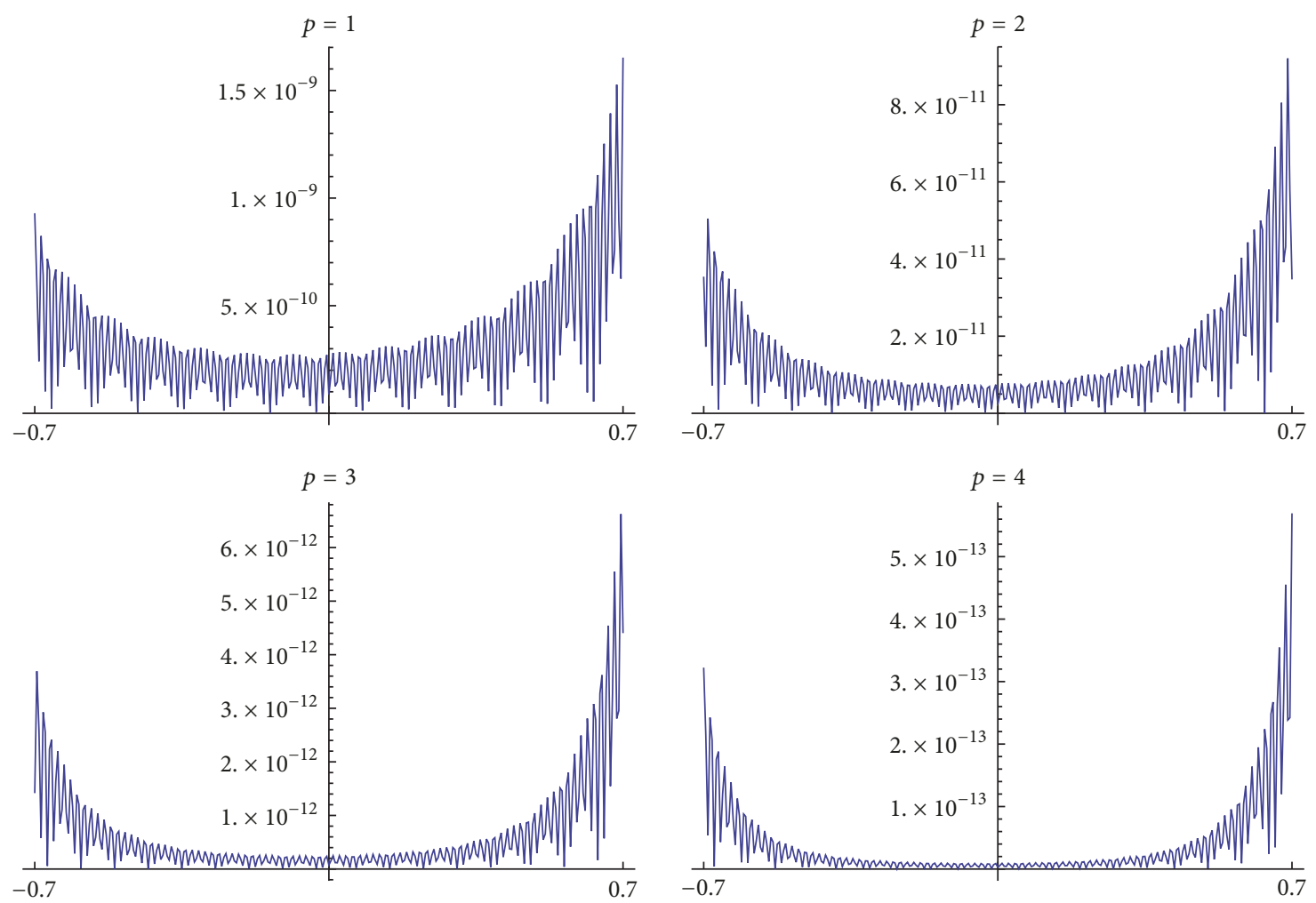

FIgURE 2: The graphs of $\left|R_{N, p}\left(f, \theta^{c}, \theta^{s}, x\right)\right|$ on $[-0.7,0.7]$ for $N=64$ and different $p$ while approximating (53) by the MTR-approximations with $\tau_{k}^{c}=\tau_{k}^{s}=k, k=1, \ldots, p$.

Lemma 8. Assume $f \in C^{2 q+p+[(p+1) / 2]+2}[-1,1]$, $f^{(2 q+p+[(p+1) / 2]+2)} \in B V[-1,1], q \geq 0, p \geq 1$, and

$$
f^{(2 k+1)}( \pm 1)=0, \quad k=0, \ldots, q-1 .
$$

Assume polynomial (60) has only real-valued and nonzero roots $x=z_{k}, k=1, \ldots, p$, and $\theta_{k}$ is defined by (26) with $\tau_{k}=z_{k}$. Let $w \leq p$, when $w$ and $p$ have the same parity, and $w \leq p+1$, otherwise. Then, the following estimates hold as $N \rightarrow \infty:$

$$
\begin{aligned}
& \Delta_{N}^{w}\left(\widehat{\Delta}^{p}\left(\theta, \widehat{f}^{c}\right)\right)=\frac{(-1)^{N}}{N^{p}} \sum_{j=0}^{r} c_{j} \sum_{t=2 q+[(p+w-j+1) / 2]}^{2 q+[(p+1) / 2]+1} \frac{1}{N^{t+2}} \\
& \cdot \sum_{s=q}^{[(t-w) / 2]} \frac{A_{2 s+1}(f)}{\pi^{2 s+2}(2 s+1) !} \sigma_{s, t, j}(w) \\
& +o\left(N^{-2 q-p-[(p+1) / 2]-3}\right), \\
& \Delta_{N}^{w}\left(\widehat{\Delta}^{p}\left(\theta, \widehat{f}^{s}\right)\right)=\frac{(-1)^{N+1}}{N p} \sum_{j=0}^{r} c_{j} \\
& \cdot \sum_{t=2 q+[(p+w-j+1) / 2]}^{2 q+[(p+1) / 2]+1} \frac{1}{N^{t+2}} \\
& \quad \sum_{s=q}^{[(t-w) / 2]} \frac{B_{2 s+1}(f)}{\pi^{2 s+2}(2 s+1) !} \widetilde{\sigma}_{s, t, j}(w)
\end{aligned}
$$

$$
+o\left(N^{-2 q-p-[(p+1) / 2]-3}\right)
$$

where

$$
\begin{aligned}
& \sigma_{s, t, j}(w)=(2 q+p \\
& +1) ! \sum_{k=0}^{p}\left(\begin{array}{l}
p \\
k
\end{array}\right) \beta_{k, s, t}(w) \frac{(p-k+t+1) !}{(2 q+p+1-k) !} k^{j}, \\
& \widetilde{\sigma}_{s, t, j}(w)=(2 q+p \\
& +1) ! \sum_{k=0}^{p}\left(\begin{array}{l}
p \\
k
\end{array}\right) \widetilde{\beta}_{k, s, t}(w) \frac{(p-k+t+1) !}{(2 q+p+1-k) !} k^{j},
\end{aligned}
$$

with $\beta$ and $\widetilde{\beta}$ defined in Lemma 5.

Proof. We prove only (64). By taking $n=N$ in (33) and using (56), we get

$$
\begin{aligned}
& \Delta_{N}^{w}\left(\widehat{\Delta}^{p}\left(\theta, \widehat{f}^{c}\right)\right)=\frac{(-1)^{N}}{N^{p}} \sum_{j=0}^{r} c_{j} \sum_{t=2 q+w}^{2 q+[(p+1) / 2]+1} \frac{1}{N^{t+2}} \\
& \quad \sum_{s=q}^{[(t-w) / 2]} \frac{A_{2 s+1}(f)}{\pi^{2 s+2}(2 s+1) !} \sigma_{s, t, j}(w) \\
& +o\left(N^{-2 q-p-[(p+1) / 2]-3}\right) .
\end{aligned}
$$

This completes the proof in view of (B.15) and (B.16). 
Further, in Theorems 9 and 10, we show that the pointwise convergence rate of the rational approximations depends on the asymptotic $\Delta_{N}^{0}\left(\widehat{\Delta}^{p}\left(\theta, \widehat{f}^{c}\right)\right)$ and $\Delta_{N}^{0}\left(\widehat{\Delta}^{p}\left(\theta, \widehat{f}^{s}\right)\right)$. From the other side, Lemma 8 reveals that the convergence rates of those sequences depend on the value of (as $w=0$ )

$$
\left[\frac{p-j+1}{2}\right] \text {. }
$$

When $p$ is odd, for the highest power of $1 / N$, parameter $j$ can be only $j=0$. It means that $Q_{r}(k) \equiv 1$. When $p$ is even, parameter $j$ can be $j \leq 1$ which means that $Q_{r}(k)=1+c_{1} k$. We determine parameter $c_{1}$ later.

Next theorem unveils the convergence rate of the MTRapproximations for odd values of $p$, when $Q_{r}(k)=Q_{0}(k) \equiv 1$. Note that, in this case, the roots of polynomial (60) coincide with the roots of generalized Laguerre polynomial $L_{p}^{(2 q+1)}(x)$ (see Appendix A).

Theorem 9. Let parameter $p \geq 1$ be odd, $f \in$ $C^{2 q+p+(p+1) / 2+2}[-1,1], q \geq 0, f^{(2 q+p+(p+1) / 2+2)} \in B V[-1,1]$, and

$$
f^{(2 k+1)}( \pm 1)=0, \quad k=0, \ldots, q-1 .
$$

Let $\theta_{k}, k=1, \ldots, p$, be defined by (26), where $\tau_{k}$ are the roots of the generalized Laguerre polynomial $L_{p}^{(2 q+1)}(x)$. Then, the following estimates hold for $|x|<1$ :

$$
\begin{aligned}
& R_{N, p}^{c}(f, \theta, x)=A_{2 q+1}(f) \\
& \cdot \frac{(-1)^{N+1}}{N^{2 q+p+(p+1) / 2+2} \pi^{2 q+2} 2^{p+1}}\left(\frac{\cos (\pi x / 2)(2 N-p+1)}{\cos ^{p+1}(\pi x / 2)}\right. \\
& \cdot \sigma_{q, 2 q+(p+1) / 2,0}(0)+\frac{\cos (\pi x / 2)(2 N-p)}{2 \cos ^{p+2}(\pi x / 2)} \\
& \left.\cdot \sigma_{q, 2 q+(p+1) / 2,0}(1)\right)+o\left(N^{-2 q-p-(p+1) / 2-2}\right), \\
& R_{N, p}^{s}(f, \theta, x)=B_{2 q+1}(f) \quad N \longrightarrow \infty, \\
& \cdot \frac{(-1)^{N}}{N^{2 q+p+(p+1) / 2+2} \pi^{2 q+2} 2^{p+1}}\left(\frac{\sin (\pi x / 2)(2 N-p)}{\cos ^{p+1}(\pi x / 2)}\right. \\
& \cdot \widetilde{\sigma}_{q, 2 q+(p+1) / 2,0}(0)+\frac{\sin (\pi x / 2)(2 N-p-1)}{2 \cos ^{p+2}(\pi x / 2)} \\
& \left.\cdot \widetilde{\sigma}_{q, 2 q+(p+1) / 2,0}(1)\right)+o\left(N^{-2 q-p-(p+1) / 2-2}\right),
\end{aligned}
$$

where $\sigma$ and $\widetilde{\sigma}$ are defined in Lemma 8.

Proof. We use (18) to estimate $R_{N, p}^{c}(f, \theta, x)$. The error $R_{N, p}^{s}(f, \theta, x)$ can be estimated similarly.

Taking into account the fact that $\theta_{k} \rightarrow 1$ as $N \rightarrow \infty$, we estimate only the sums on the right-hand side of (18).
An application of the Abel transformation to the sums of $R_{N, p}^{c}(f, \theta, x)$ leads to the following expansion of the error:

$$
\begin{aligned}
& \sum_{n=N+1}^{\infty} \Delta_{n}^{p}\left(\theta, \widehat{f}^{c}\right) e^{ \pm i \pi n x}=-\frac{e^{ \pm i \pi(N+1) x}}{\left(1+e^{ \pm i \pi x}\right)} \Delta_{N}^{p}\left(\theta, \widehat{f}^{c}\right) \\
& -\frac{e^{ \pm i \pi(N+1) x}}{\left(1+e^{ \pm i \pi x}\right)^{2}} \Delta_{N}^{1}\left(\widehat{\Delta}^{p}\left(\theta, \widehat{f}^{c}\right)\right) \\
& -e^{ \pm i \pi(N+1) x} \sum_{w=2}^{(p+1) / 2+1} \frac{\Delta_{N}^{w}\left(\widehat{\Delta}^{p}\left(\theta, \widehat{f}^{c}\right)\right)}{\left(1+e^{ \pm i \pi x}\right)^{w+1}} \\
& +\frac{1}{\left(1+e^{ \pm i \pi x}\right)^{(p+1) / 2+2}} \\
& \cdot \sum_{n=N+1}^{\infty} \Delta_{n}^{(p+1) / 2+2}\left(\widehat{\Delta}^{p}\left(\theta, \widehat{f}^{c}\right)\right) e^{ \pm i \pi n x} .
\end{aligned}
$$

According to Lemma 5, we have

$$
\begin{aligned}
\Delta_{n}^{(p+1) / 2+2}\left(\widehat{\Delta}^{p}\left(\theta, \widehat{f}^{c}\right)\right)=\frac{o\left(N^{-p}\right)}{n^{2 q+(p+1) / 2+3}}, & \\
& N \longrightarrow \infty, n \geq N+1,
\end{aligned}
$$

and hence the last term on the right-hand side of (71) is $o\left(N^{-2 q-p-(p+1) / 2-2}\right)$ as $N \rightarrow \infty$. It follows from Lemma 8 that the third term in (71) is $O\left(N^{-2 q-p-(p+1) / 2-3}\right)$ as $N \rightarrow \infty$. Therefore,

$$
\begin{aligned}
& R_{N, p}^{c}(f, \theta, x) \\
& =-\left(\frac{e^{i \pi(N+1) x}}{2\left(1+e^{i \pi x}\right)^{p+1}}+\frac{e^{-i \pi(N+1) x}}{2\left(1+e^{-i \pi x}\right)^{p+1}}\right) \\
& \cdot \Delta_{N}^{p}\left(\theta, \hat{f}^{c}\right) \\
& \quad-\left(\frac{e^{i \pi(N+1) x}}{2\left(1+e^{i \pi x}\right)^{p+2}}+\frac{e^{-i \pi(N+1) x}}{2\left(1+e^{-i \pi x}\right)^{p+2}}\right) \\
& \cdot \Delta_{N}^{1}\left(\widehat{\Delta}^{p}\left(\theta, \widehat{f}^{c}\right)\right)+o\left(N^{-2 q-p-p / 2-2}\right) .
\end{aligned}
$$

By Lemma 8 , we have

$$
\begin{aligned}
& \Delta_{N}^{p}\left(\theta, \widehat{f}^{c}\right)=\frac{(-1)^{N}}{N^{p}} \\
& \cdot \sum_{t=2 q+(p+1) / 2}^{2 q+(p+1) / 2+1} \frac{1}{N^{t+2}} \sum_{s=q}^{[t / 2]} \frac{A_{2 s+1}(f)}{\pi^{2 s+2}} \sigma_{s, t, 0}(0) \\
& +o\left(N^{-2 q-p-(p+1) / 2-3}\right)=\frac{(-1)^{N}}{(\pi N)^{2 q+2} N^{p+(p+1) / 2}} \\
& \quad \cdot \sum_{s=q}^{q+[(p+1) / 4]} \frac{A_{2 s+1}(f)}{\pi^{2 s}} \sigma_{s, 2 q+(p+1) / 2,0}(0) \\
& \quad+o\left(N^{-2 q-p-(p+1) / 2-2}\right), \\
& \Delta_{N}^{1}\left(\widehat{\Delta}^{p}\left(\theta, \widehat{f}^{c}\right)\right)=\frac{(-1)^{N}}{N^{p}} \sum_{t=2 q+(p+1) / 2}^{2 q+(p+1) / 2+1} \frac{1}{N^{t+2}}
\end{aligned}
$$




$$
\begin{aligned}
& \cdot \sum_{s=q}^{[(t-1) / 2]} \frac{A_{2 s+1}(f)}{\pi^{2 s+2}} \sigma_{s, t, 0}(1)+o\left(N^{-2 q-p-(p+1) / 2-3}\right) \\
& =\frac{(-1)^{N}}{(\pi N)^{2 q+2} N^{p+(p+1) / 2}} \\
& \cdot \sum_{s=q}^{q+[(p-1) / 4]} \frac{A_{2 s+1}(f)}{\pi^{2 s}} \sigma_{s, 2 q+(p+1) / 2,0}(1) \\
& +o\left(N^{-2 q-p-(p+1) / 2-2}\right) .
\end{aligned}
$$

Taking into account the fact that (see (B.16))

$$
\sigma_{s, 2 q+(p+1) / 2,0}(0)=\sigma_{s, 2 q+(p+1) / 2,0}(1)=0, \quad s>q,
$$

we conclude that $\sigma_{s, 2 q+(p+1) / 2,0}(0)$ and $\sigma_{s, 2 q+(p+1) / 2,0}(1)$ are nonzero only for $s=q$ which leads to the following estimates:

$$
\begin{aligned}
\Delta_{N}^{p}\left(\theta, \widehat{f}^{c}\right) & \\
= & A_{2 q+1}(f) \frac{(-1)^{N}}{(\pi N)^{2 q+2} N^{p+(p+1) / 2}} \sigma_{q, 2 q+(p+1) / 2,0}(0) \\
& \quad+o\left(N^{-2 q-p-(p+1) / 2-2}\right), \\
\Delta_{N}^{1}\left(\widehat{\Delta}^{p}\left(\theta, \widehat{f}^{c}\right)\right) & \\
= & A_{2 q+1}(f) \frac{(-1)^{N}}{(\pi N)^{2 q+2} N^{p+(p+1) / 2}} \sigma_{q, 2 q+(p+1) / 2,0}(1) \\
& +o\left(N^{-2 q-p-(p+1) / 2-2}\right) .
\end{aligned}
$$

From here, we get

$$
\begin{aligned}
& R_{N, p}^{c}(f, \theta, x)=A_{2 q+1}(f) \\
& \cdot \frac{(-1)^{N+1}}{\pi^{2 q+2} N^{2 q+p+p / 2+2}}\left(\sigma_{q, 2 q+(p+1) / 2,0}(0)\right. \\
& \quad \cdot \operatorname{Re}\left[\frac{e^{i \pi(N+1) x}}{\left(1+e^{i \pi x}\right)^{p+1}}\right]+\sigma_{q, 2 q+(p+1) / 2,0}(1)
\end{aligned}
$$

$$
\left.\cdot \operatorname{Re}\left[\frac{e^{i \pi(N+1) x}}{\left(1+e^{i \pi x}\right)^{p+2}}\right]\right)+o\left(N^{-2 q-p-(p+1) / 2-2}\right)
$$

which completes the proof.

Figure 3 shows the result of approximation of (53) by the MTR-approximations with optimal parameters $\tau_{k}^{c}=\tau_{k}^{s}, k=$ $1, \ldots, p$ as the roots of $L_{p}^{(2 q+1)}(x)$. We see better accuracy on $[-0.7,0.7]$ compared to nonoptimal parameters as in Figure 2. For $p=1$, the improvement is almost 25 times, and for $p=3$, the improvement is almost 240 times.

Next theorem deals with even values of $p$. As we mentioned above, the best convergence rate is possible if $Q_{r}(k)=$ $Q_{1}(k)=1+c_{1} k$ and $\tau_{k}, k=1, \ldots, p$, are the roots of $(60)$. We need to assume that polynomial (60) has only real-valued and nonzero roots $x=z_{k}, k=1, \ldots, p$, for fixed $p$ and $q$. In two cases, we can prove that it is true. When $c_{1}=0$, the roots of polynomial (60) coincide with the roots of the generalized Laguerre polynomial $L_{p}^{(2 q+1)}(x)$ (see Appendix A). When $c_{1}=-1 /(2 q+1+p)$, the roots coincide with the ones of $L_{p}^{(2 q)}(x)$.

We saw from our experiments (which we cannot prove theoretically) that polynomial (60) has only real-valued and nonzero roots also for other values of parameter $c_{1}$. However, based on our experiments, we observed that the rational approximations have almost similar accuracy for different values of $c_{1}$ while approximating smooth functions on $|x|<1$.

Theorem 10. Let parameter $p \geq 2$ be even, $f \in$ $C^{2 q+p+p / 2+2}[-1,1], q \geq 0, f^{(2 q+p+p / 2+2)} \in B V[-1,1]$, and

$$
f^{(2 k+1)}( \pm 1)=0, \quad k=0, \ldots, q-1 .
$$

Assume the polynomial

$$
\sum_{k=0}^{p}\left(\begin{array}{l}
p \\
k
\end{array}\right) \frac{1+c_{1}(p-k)}{(2 q+1+k) !}(-1)^{k} x^{k}
$$

has only real-valued and nonzero roots $x=z_{k}, k=1, \ldots, p$, and let $\theta_{k}$ be defined by (26) with $\tau_{k}=z_{k}$. Then, the following estimates hold for $|x|<1$ :

$$
\begin{aligned}
& R_{N, p}^{c}(f, \theta, x)=A_{2 q+1}(f) \frac{(-1)^{N+1}}{N^{2 q+p+p / 2+2} \pi^{2 q+2} 2^{p+1}}\left(\frac{\cos (\pi x / 2)(2 N-p+1)}{\cos ^{p+1}(\pi x / 2)}\left(\sigma_{q, 2 q+p / 2,0}(0)+\sigma_{q, 2 q+p / 2,1}(0) c_{1}\right)\right. \\
& \left.+\frac{\cos (\pi x / 2)(2 N-p) \sigma_{q, 2 q+p / 2,1}(1)}{2 \cos ^{p+2}(\pi x / 2)} c_{1}\right)+o\left(N^{-2 q-p-p / 2-2}\right), \quad N \rightarrow \infty, \\
& R_{N, p}^{s}(f, \theta, x)=B_{2 q+1}(f) \frac{(-1)^{N}}{N^{2 q+p+p / 2+2} \pi^{2 q+2} 2^{p+1}}\left(\frac{\sin (\pi x / 2)(2 N-p)}{\cos ^{p+1}(\pi x / 2)}\left(\widetilde{\sigma}_{q, 2 q+p / 2,0}(0)+\widetilde{\sigma}_{q, 2 q+p / 2,1}(0) c_{1}\right)\right. \\
& \left.+\frac{\sin (\pi x / 2)(2 N-p-1) \widetilde{\sigma}_{q, 2 q+p / 2,1}(1)}{2 \cos ^{p+2}(\pi x / 2)} c_{1}\right)+o\left(N^{-2 q-p-p / 2-2}\right), \quad N \rightarrow \infty,
\end{aligned}
$$

where $\sigma$ and $\widetilde{\sigma}$ are defined in Lemma 8. 

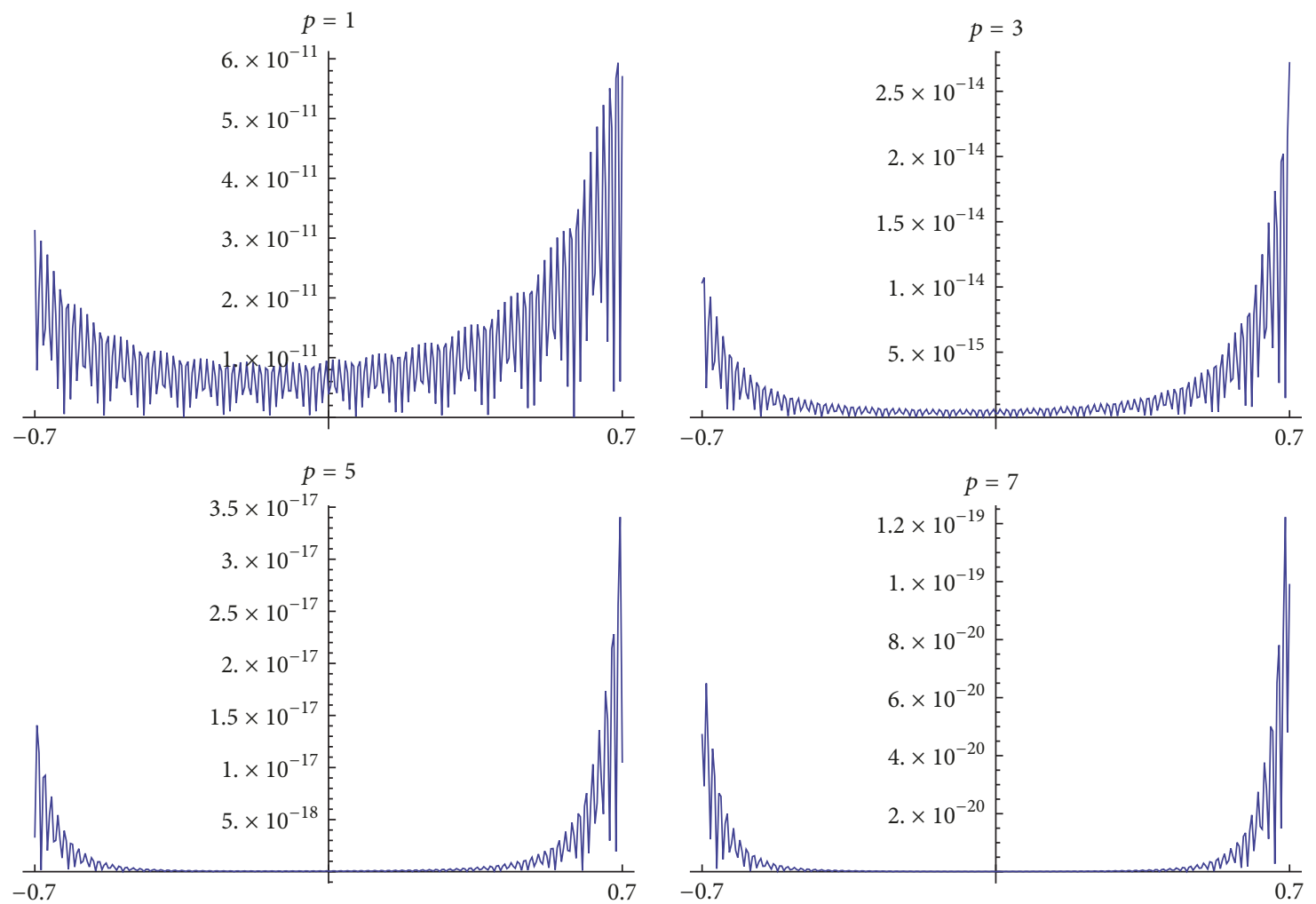

Figure 3: The graphs of $\left|R_{N, p}\left(f, \theta^{c}, \theta^{s}, x\right)\right|$ on interval [-0.7,0.7] for $N=64$ while approximating (53) by MTR-approximations. Parameters $\tau_{k}^{c}$ and $\tau_{k}^{s}, k=1, \ldots, p$, are the roots of $L_{p}^{(2 q+1)}(x)$ which are optimal for odd $p$ on $|x|<1$ (see Theorem 9).

Proof. We prove only (80) and need only to estimate the sums on the right-hand side of (18). Similar to (71), we apply the Abel transformation and get the following expansion of the error:

$$
\begin{aligned}
& \sum_{n=N+1}^{\infty} \Delta_{n}^{p}\left(\theta, \widehat{f}^{c}\right) e^{ \pm i \pi n x} \\
= & -\frac{e^{ \pm i \pi(N+1) x}}{\left(1+e^{ \pm i \pi x}\right)} \Delta_{N}^{p}\left(\theta, \widehat{f}^{c}\right) \\
& -\frac{e^{ \pm i \pi(N+1) x}}{\left(1+e^{ \pm i \pi x}\right)^{2}} \Delta_{N}^{1}\left(\widehat{\Delta}^{p}\left(\theta, \widehat{f}^{c}\right)\right) \\
& -e^{ \pm i \pi(N+1) x} \sum_{w=2}^{p / 2+1} \frac{\Delta_{N}^{w}\left(\widehat{\Delta}^{p}\left(\theta, \widehat{f}^{c}\right)\right)}{\left(1+e^{ \pm i \pi x}\right)^{w+1}} \\
& +\frac{1}{\left(1+e^{ \pm i \pi x}\right)^{p / 2+2}} \sum_{n=N+1}^{\infty} \Delta_{n}^{p / 2+2}\left(\widehat{\Delta}^{p}\left(\theta, \widehat{f}^{c}\right)\right) e^{ \pm i \pi n x} .
\end{aligned}
$$

Taking into account Lemma 5, we obtain

$$
\begin{aligned}
& \Delta_{n}^{p / 2+2}\left(\widehat{\Delta}^{p}\left(\theta, \widehat{f}^{c}\right)\right)=\frac{o\left(N^{-p}\right)}{n^{2 q+p / 2+3}}, \\
& \quad N \longrightarrow \infty, n \geq N+1 .
\end{aligned}
$$

And the last term on the right-hand side of (82) is $o\left(N^{-2 q-p-p / 2-2}\right)$ as $N \rightarrow \infty$. According to Lemma 8 , the third term in the right-hand side of (82) is $O\left(N^{-2 q-p-p / 2-3}\right)$ as $N \rightarrow \infty$. Therefore,

$$
\begin{aligned}
R_{N, p}^{c} & (f, \theta, x) \\
= & -\left(\frac{e^{i \pi(N+1) x}}{2\left(1+e^{i \pi x}\right)^{p+1}}+\frac{e^{-i \pi(N+1) x}}{2\left(1+e^{-i \pi x}\right)^{p+1}}\right) \\
& \cdot \Delta_{N}^{p}\left(\theta, \widehat{f}^{c}\right) \\
& -\left(\frac{e^{i \pi(N+1) x}}{2\left(1+e^{i \pi x}\right)^{p+2}}+\frac{e^{-i \pi(N+1) x}}{2\left(1+e^{-i \pi x}\right)^{p+2}}\right) \\
& \cdot \Delta_{N}^{1}\left(\widehat{\Delta}^{p}\left(\theta, \widehat{f}^{c}\right)\right)+o\left(N^{-2 q-p-p / 2-2}\right) .
\end{aligned}
$$

According to Lemma 8, we get

$$
\begin{aligned}
& \Delta_{N}^{p}\left(\theta, \widehat{f}^{c}\right)=\frac{(-1)^{N}}{N^{p}} \sum_{j=0}^{1} c_{j} \sum_{t=2 q+p / 2}^{2 q+p / 2+1} \frac{1}{N^{t+2}} \\
& \cdot \sum_{s=q}^{[t / 2]} \frac{A_{2 s+1}(f)}{\pi^{2 s+2}} \sigma_{s, t, j}(0)+o\left(N^{-2 q-p-p / 2-3}\right) \\
& =\frac{(-1)^{N}}{(\pi N)^{2 q+2} N^{p+p / 2}} \sum_{j=0}^{1} c_{j}
\end{aligned}
$$




$$
\begin{aligned}
& \cdot \sum_{s=q}^{q+[p / 4]} \frac{A_{2 s+1}(f)}{\pi^{2 s}} \sigma_{s, 2 q+p / 2, j}(0) \\
& +o\left(N^{-2 q-p-p / 2-2}\right), \\
\Delta_{N}^{1} & \left(\widehat{\Delta}^{p}\left(\theta, \widehat{f}^{c}\right)\right)=\frac{(-1)^{N}}{N^{p}} \sum_{j=0}^{1} c_{j} \sum_{t=2 q+[(p+2-j) / 2]}^{2 q+p / 2+1} \frac{1}{N^{t+2}} \\
& \cdot \sum_{s=q}^{[(t-1) / 2]} \frac{A_{2 s+1}(f)}{\pi^{2 s+2}} \sigma_{s, t, j}(1)+o\left(N^{-2 q-p-p / 2-3}\right) \\
& =\frac{(-1)^{N} c_{1}}{(\pi N)^{2 q+2} N^{p+p / 2}} \\
& \cdot \sum_{s=q}^{q+[(p-2) / 4]} \frac{A_{2 s+1}(f)}{\pi^{2 s}} \sigma_{s, 2 q+p / 2,1}(1) \\
& +o\left(N^{-2 q-p-p / 2-2}\right) .
\end{aligned}
$$

According to (B.16), we have

$$
\begin{aligned}
& \sigma_{s, 2 q+(p+1) / 2, j}(0)=\sigma_{s, 2 q+(p+1) / 2, j}(0)=0, \quad s>q, \\
& \sigma_{s, 2 q+(p+1) / 2,1}(1)=0, \quad s>q .
\end{aligned}
$$

Hence, $\sigma_{s, 2 q+(p+1) / 2, j}(0), j=0,1$ and $\sigma_{s, 2 q+(p+1) / 2,1}(1)$ are nonzero only for $s=q$, which leads to the following estimates:

$$
\begin{aligned}
& \Delta_{N}^{p}\left(\theta, \widehat{f}^{c}\right)=A_{2 q+1}(f) \\
& \cdot \frac{(-1)^{N}}{(\pi N)^{2 q+2} N^{p+p / 2}}\left(\sigma_{q, 2 q+p / 2,0}(0)\right. \\
& \left.+\sigma_{q, 2 q+p / 2,1}(0) c_{1}\right)+o\left(N^{-2 q-p-p / 2-2}\right), \\
& \Delta_{N}^{1}\left(\widehat{\Delta}^{p}\left(\theta, \widehat{f}^{c}\right)\right)=A_{2 q+1}(f) \frac{(-1)^{N} c_{1}}{(\pi N)^{2 q+2} N^{p+p / 2}} \\
& \cdot \sigma_{q, 2 q+p / 2,1}(1)+o\left(N^{-2 q-p-p / 2-2}\right) .
\end{aligned}
$$

Finally, from (84), we get

$$
\begin{aligned}
& R_{N, p}^{c}(f, \theta, x)=A_{2 q+1}(f) \\
& \cdot \frac{(-1)^{N+1}}{\pi^{2 q+2} N^{2 q+p+p / 2+2}}\left(\left(\sigma_{q, 2 q+p / 2,0}(0)\right.\right. \\
& \left.+\sigma_{q, 2 q+p / 2,1}(0) c_{1}\right) \operatorname{Re}\left[\frac{e^{i \pi(N+1) x}}{\left(1+e^{i \pi x}\right)^{p+1}}\right] \\
& \left.\quad+\sigma_{q, 2 q+p / 2,1}(1) \operatorname{Re}\left[\frac{e^{i \pi(N+1) x}}{\left(1+e^{i \pi x}\right)^{p+2}}\right] c_{1}\right) \\
& \quad+o\left(N^{-2 q-p-p / 2-2}\right),
\end{aligned}
$$

which completes the proof.
Theorems 9 and 10 conclude that by appropriate determination of parameters $\tau_{k}^{c}$ and $\tau_{k}^{s}, k=1, \ldots, p$, we get extra improvement of the convergence rate of the MTRapproximations by factor $O\left(N^{[(p+1) / 2]}\right)$ compared to Theorem 6. Final improvement compared to the modified expansion is by factor $O\left(N^{p+[(p+1) / 2]}\right)$.

Let us return to MTR-approximations of (53). Figure 4 shows the results of approximation of (53) by the MTRapproximations with even $p$. Parameters $\tau_{k}^{c}=\tau_{k}^{s}, k=$ $1, \ldots, p$ are selected as the roots of $L_{p}^{(2 q+1)}(x)$. Compared with Figure 2, we see better accuracy on $|x|<1$. For $p=2$, the improvement is almost 27 times, and for $p=4$, the improvement is almost 200 times.

Figure 5 shows similar results with parameters $\tau_{k}^{c}=\tau_{k}^{s}$, $k=1, \ldots, p$, as the roots of $L_{p}^{(2 q)}(x)$. In the next section, we will prove that those parameters provide improved accuracy also at $x= \pm 1$ for some $p$ and $q$. We see that both choices of parameters provide similar results on $|x|<1$.

\section{Pointwise Convergence at the Endpoints}

In this section, we explore the pointwise convergence of the MTR-approximations at the endpoints $x= \pm 1$. Next theorem explores the convergence of the rational approximations without determining parameters $\tau^{c}$ and $\tau^{s}$.

Theorem 11. Assume $f \in C^{2 q+p+1}[-1,1], f^{(2 q+p+1)} \in B V[-1$, $1], q \geq 0, p \geq 1$, and

$$
f^{(2 k+1)}( \pm 1)=0, \quad k=0, \ldots, q-1 .
$$

Let $\theta_{k}, k=1, \ldots, p$ be defined by (26). Then, the following estimates hold:

$$
\begin{aligned}
R_{N, p}^{c} & (f, \theta, \pm 1) \\
= & \frac{A_{2 q+1}(f)}{N^{2 q+1}} \frac{(-1)^{p}}{(2 q+1) ! \pi^{2 q+2} \gamma_{p}(\tau)} h_{p, 2 q}(\tau) \\
& +o\left(N^{-2 q-1}\right), \quad N \longrightarrow \infty, \\
R_{N, p}^{s} & (f, \theta, \pm 1) \\
= & \pm \frac{B_{2 q+1}(f)}{N^{2 q+1}} \frac{(-1)^{p}}{(2 q+1) ! \pi^{2 q+2} \gamma_{p}(\tau)} h_{p, 2 q}(\tau) \\
& +o\left(N^{-2 q-1}\right), \quad N \longrightarrow \infty,
\end{aligned}
$$

where $h_{p, m}(\tau)$ is defined by $(46)$.

Proof. In view of (18) and (19), we write

$$
\begin{aligned}
& R_{N, p}^{c}(f, \theta, \pm 1)=\frac{N^{p}}{\gamma_{p}(\tau)} \sum_{n=N+1}^{\infty} \Delta_{n}^{p}\left(\theta, \hat{f}^{c}\right)(-1)^{n}, \\
& R_{N, p}^{s}(f, \theta, \pm 1)=\mp \frac{N^{p}}{\gamma_{p}(\tau)} \sum_{n=N+1}^{\infty} \Delta_{n}^{p}\left(\theta, \hat{f}^{s}\right)(-1)^{n} .
\end{aligned}
$$



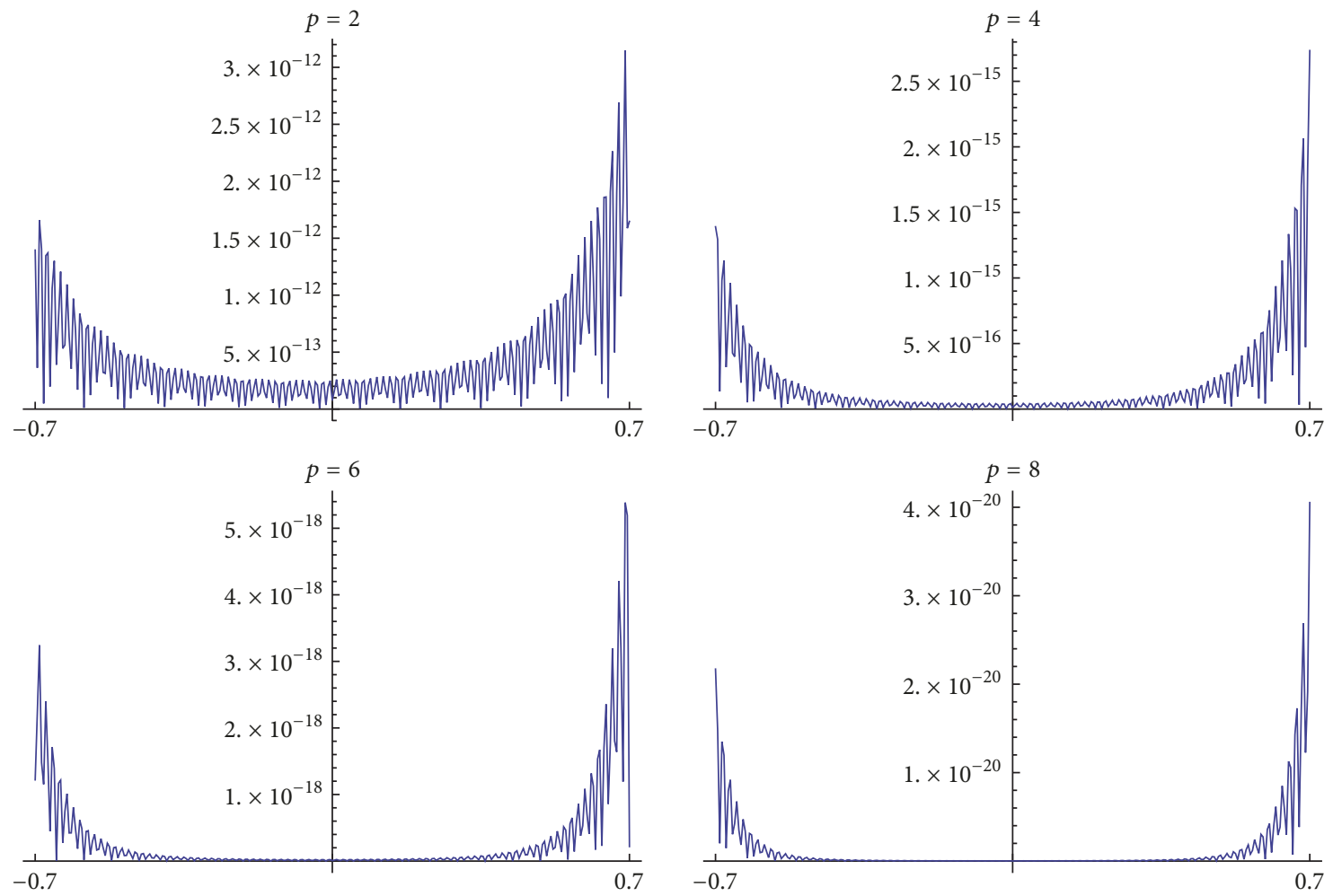

FIGURE 4: The graphs of $\left|R_{N, p}\left(f, \theta^{c}, \theta^{s}, x\right)\right|$ on interval $[-0.7,0.7]$ for $N=64$ while approximating (53). Parameters $\tau_{k}^{c}$ and $\tau_{k}^{s}, k=1, \ldots, p$, are the roots of $L_{p}^{(2 q+1)}(x)$.
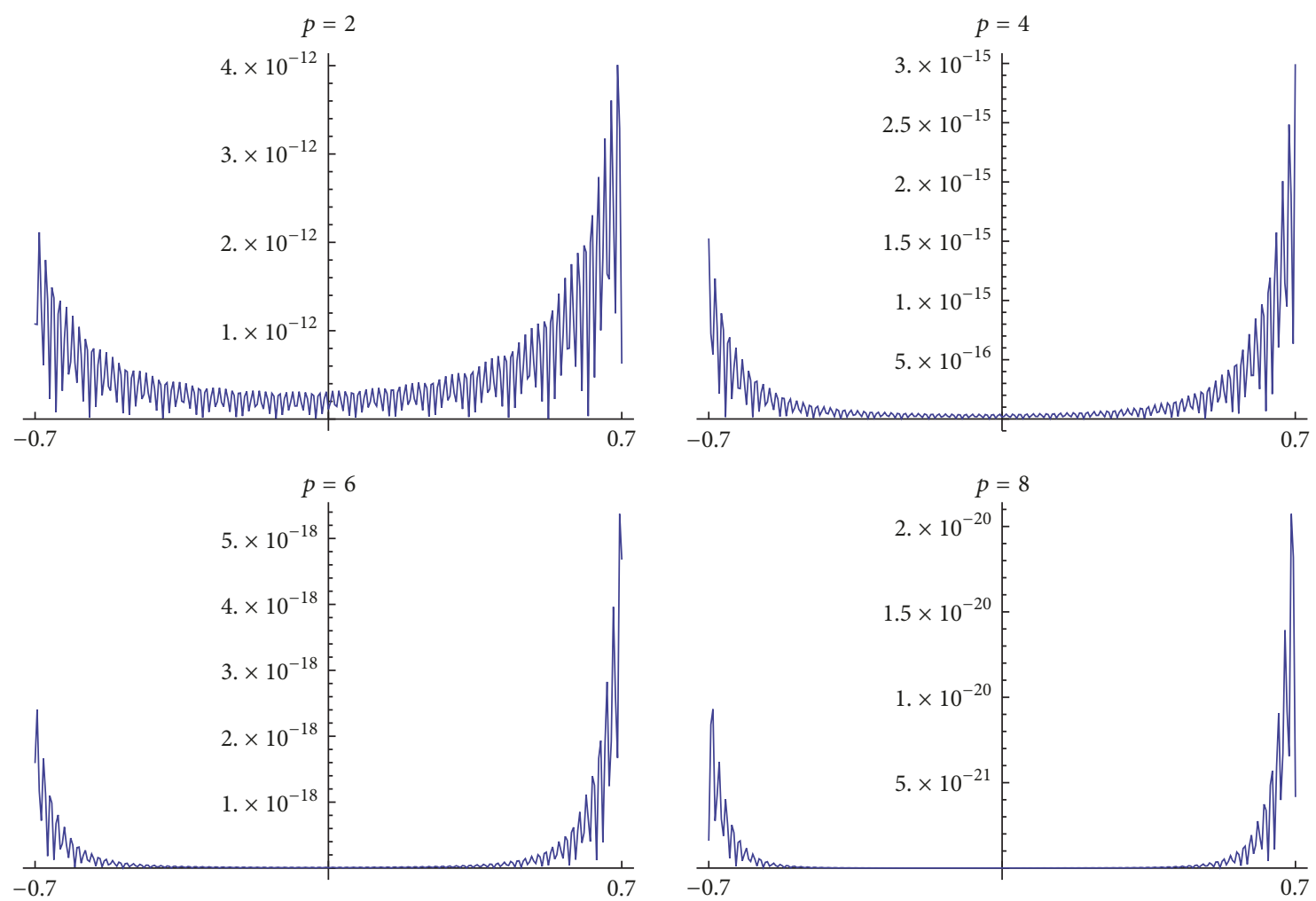

Figure 5: The graphs of $\left|R_{N, p}\left(f, \theta^{c}, \theta^{s}, x\right)\right|$ on interval [-0.7,0.7] for $N=64$ while approximating (53). Parameters $\tau_{k}^{c}$ and $\tau_{k}^{s}, k=1, \ldots, p$, are the roots of $L_{p}^{(2 q)}(x)$. 
Now, the proof immediately follows from the estimates of Lemma 5 by taking $w=0, r=0$ and by recalling that $\alpha_{p-k, p-k}=(-1)^{p-k}(p-k)$ !.

Note that this theorem is valid also for $p=0$ which corresponds to the expansions by the modified Fourier basis (compare with Theorems 1 and 2).

Exact constants of the main terms in (90), for $p=0$, can be found also in [10] (Theorem 3.2). We see that, in general, rational corrections do not increase the convergence rates of modified Fourier expansions at the endpoints $x= \pm 1$ without specifying appropriately parameters $\tau^{c}$ and $\tau^{s}$. Both approaches have the same convergence rates $O\left(N^{-2 q-1}\right)$.

Moreover, as Figure 6 shows, without reasonable selection of the parameters, modified Fourier expansions have better accuracy compared to "nonoptimal" rational approximations at $x= \pm 1$.

Is it possible to improve the accuracy by appropriate selection of parameters $\tau^{c}$ and $\tau^{s}$ ? The answer is positive and the solution is in the estimates of Theorem 11. Like the previous section, we put

$$
\gamma_{k}(\tau)=\left(\begin{array}{l}
p \\
k
\end{array}\right) \frac{(2 q+p) !}{(2 q+p-k) !} P_{r}(k),
$$

where

$$
P_{r}(k)=\sum_{j=0}^{r} d_{j} k^{j}, \quad d_{0}=1 .
$$

Now, the property $h_{p, 2 q}(\tau)=0$ follows from the identity

$$
\sum_{k=0}^{p}(-1)^{k}\left(\begin{array}{l}
p \\
k
\end{array}\right) k^{j}=0, \quad j<p .
$$

Next theorem is the result of these observations and Theorem 11.

Theorem 12. Let $f \in C^{2 q+p+1}[-1,1], q \geq 0, p \geq 1, f^{(2 q+p+1)} \in$ $B V[-1,1]$, and

$$
f^{(2 k+1)}( \pm 1)=0, \quad k=0, \ldots, q-1 .
$$

Assume the polynomial

$$
\sum_{k=0}^{p}\left(\begin{array}{l}
p \\
k
\end{array}\right) \frac{P_{r}(p-k)}{(2 q+k) !}(-1)^{k} x^{k}
$$

has only real-valued and nonzero roots $x=z_{k}, k=1, \ldots, p$, and let

$$
\theta_{k}^{c}=\theta_{k}^{s}=1-\frac{z_{k}}{N}, \quad k=1, \ldots, p
$$

Then,

$$
R_{N, p}\left(f, \theta^{c}, \theta^{s}, \pm 1\right)=o\left(N^{-2 q-1}\right), \quad N \longrightarrow \infty .
$$

Note that Theorem 12 is valid only for those parameters $p$ and $q$ when polynomial (97) has only real-valued and nonzero roots. Further, we clarify this property with more details.

Our next goal is derivation of the exact convergence rate of (99).
Lemma 13. Assume $f \in C^{2 q+p+[(p+1) / 2]+1}[-1,1], q \geq 0, p \geq 1$, $f^{(2 q+p+[(p+1) / 2]+1)} \in B V[-1,1]$, and

$$
f^{(2 k+1)}( \pm 1)=0, \quad k=0, \ldots, q-1 .
$$

Assume the polynomial

$$
\sum_{k=0}^{p}\left(\begin{array}{l}
p \\
k
\end{array}\right) \frac{P_{r}(p-k)}{(2 q+k) !}(-1)^{k} x^{k}
$$

has only real-valued and nonzero roots $x=z_{k}, k=1, \ldots, p$, and let $\theta_{k}$ be defined by (26) with $\tau_{k}=z_{k}$. Then, the following asymptotic expansions hold:

$$
\begin{aligned}
& R_{N, p}^{c}(f, \theta, \pm 1)=\frac{1}{\gamma_{p}(\tau)} \sum_{j=0}^{r} d_{j} \sum_{t=2 q+[(p+1-j) / 2]}^{2 q+[(p+1) / 2]} \frac{1}{N^{t+1}} \\
& \cdot\left(\sum_{\ell=0}^{t-2 q} b_{\ell} \frac{(-1)^{\ell}}{\ell !} \sum_{s=q}^{[(t-\ell) / 2]} \frac{A_{2 s+1}(f)}{\pi^{2 s+2}(2 s+1) !} \delta_{q, t-\ell, j}(\ell)\right. \\
& \left.-\sum_{s=q}^{[t / 2]} \frac{A_{2 s+1}(f)}{\pi^{2 s+2}(2 s+1) !} \delta_{s, t, j}(1)\right) \\
& +o\left(N^{-2 q-[(p+1) / 2]-1}\right), \quad N \longrightarrow \infty, \\
& R_{N, p}^{s}(f, \theta, \pm 1)= \pm \frac{1}{\gamma_{p}(\tau)} \sum_{j=0}^{r} d_{j} \sum_{t=2 q+[(p+1-j) / 2]}^{2 q+[(p+1) / 2]} \frac{1}{N^{t+1}} \\
& \cdot\left(\sum_{\ell=0}^{t-2 q} b_{\ell} \frac{(-1)^{\ell}}{\ell !} \sum_{s=q}^{[(t-\ell) / 2]} \frac{B_{2 s+1}(f)}{\pi^{2 s+2}(2 s+1) !} \widetilde{\delta}_{q, t-\ell, j}(\ell)\right. \\
& \left.-\sum_{s=q}^{[t / 2]} \frac{B_{2 s+1}(f)}{\pi^{2 s+2}(2 s+1) !} \widetilde{\delta}_{s, t, j}(1)\right) \\
& +o\left(N^{-2 q-[(p+1) / 2]-1}\right), \quad N \longrightarrow \infty,
\end{aligned}
$$

where $b_{\ell}$ is the eth Bernoulli number and

$$
\begin{aligned}
& \delta_{s, t, j}(w) \\
& =(2 q+p) ! \sum_{k=0}^{p}\left(\begin{array}{l}
p \\
k
\end{array}\right) \beta_{k, s, t}(0) \frac{(p-k+t+w) !}{(2 q+p-k) !} k^{j}, \\
& \widetilde{\delta}_{s, t, j}(w) \\
& =(2 q+p) ! \sum_{k=0}^{p}\left(\begin{array}{l}
p \\
k
\end{array}\right) \widetilde{\beta}_{k, s, t}(0) \frac{(p-k+t+w) !}{(2 q+p-k) !} k^{j},
\end{aligned}
$$

with $\beta$ and $\widetilde{\beta}$ defined in Lemma 5. 


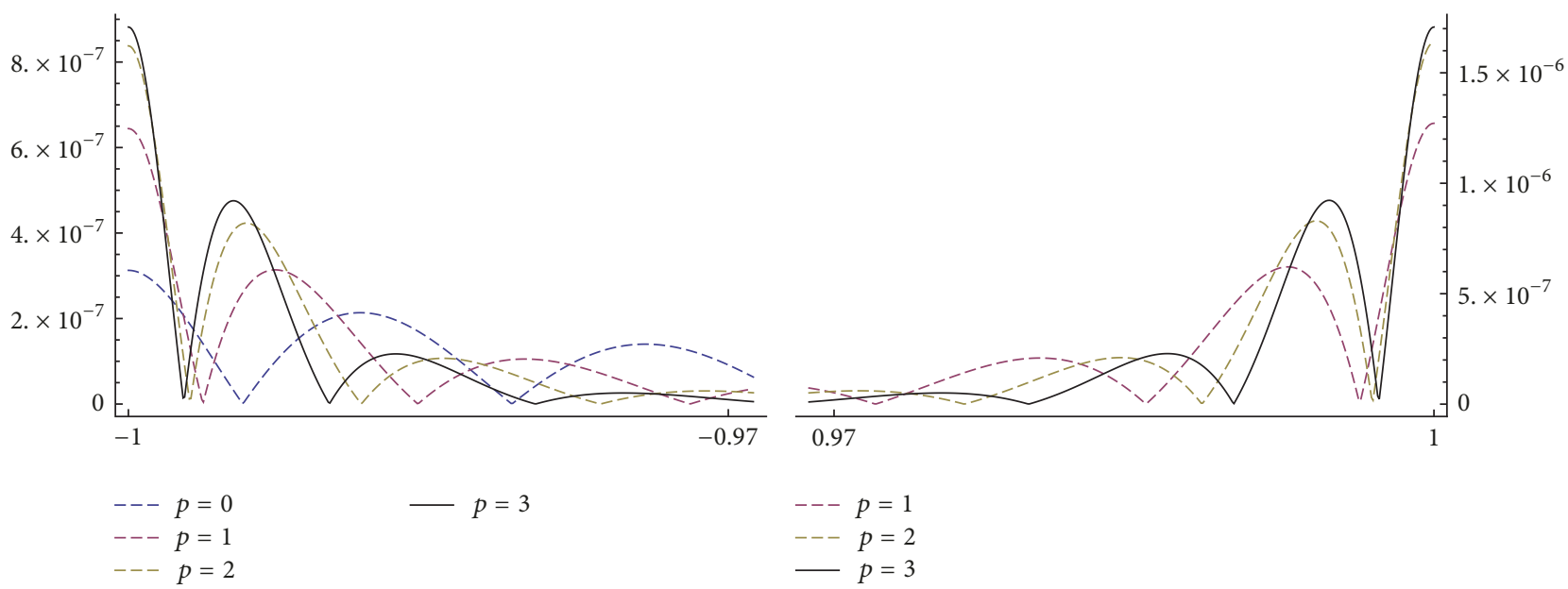

Figure 6: The graphs of $\left|R_{N, p}\left(f, \theta^{c}, \theta^{s}, x\right)\right|$ for $p=0,1,2,3$ and $N=64$ while approximating (53) at the points $x= \pm 1$. In rational approximations, we took $\tau_{k}^{c}=\tau_{k}^{s}=k, k=1, \ldots, p$.

Proof. We prove only (102). In view of Lemma 5 (with $w=0$ ) and (91), we write

$$
\begin{aligned}
R_{N, p}^{c}(f, \theta, \pm 1)= & \frac{N^{p}}{\gamma_{p}(\tau)} \sum_{k=0}^{p} \frac{\gamma_{k}(\tau)}{N^{k}} \\
& \cdot \sum_{t=2 q}^{2 q+[(p+1) / 2]}(p-k+t+1) ! \\
& \cdot \sum_{s=q}^{[t / 2]} \frac{A_{2 s+1}(f)}{\pi^{2 s+2}(2 s+1) !} \beta_{k, s, t}(0) \\
& \cdot \sum_{n=N+1}^{\infty} \frac{1}{n^{p-k+t+2}} \\
& +o\left(N^{-2 q-[(p+1) / 2]-1}\right) .
\end{aligned}
$$

We estimate the infinite sum on the right-hand side of (106) by the Euler-Maclaurin formula (see [21]). We have

$$
\begin{aligned}
& \sum_{n=N}^{\infty} \frac{1}{n^{p-k+t+2}}=\frac{1}{p-k+t+1} \\
& \quad \sum_{w=0}^{2 q+[(p+1) / 2]-t}\left(\begin{array}{c}
p-k+t+w \\
w
\end{array}\right) \frac{b_{w}(-1)^{w}}{N^{p-k+t+w+1}} \\
& \quad+O\left(N^{-2 q-p+k-[(p+1) / 2]-2}\right),
\end{aligned}
$$

where $b_{w}$ is the $w$ th Bernoulli number. Then,

$$
\begin{aligned}
R_{N, p}^{c}(f, \theta, \pm 1)= & \frac{1}{\gamma_{p}(\tau)} \sum_{j=0}^{r} d_{j} \sum_{t=2 q}^{2 q+[(p+1) / 2]} \frac{1}{N^{t+1}} \\
& \cdot \sum_{\ell=0}^{t-2 q} b_{\ell} \frac{(-1)^{\ell}}{\ell !}
\end{aligned}
$$

$$
\begin{aligned}
\cdot & \sum_{s=q}^{[(t-\ell) / 2]} \frac{A_{2 s+1}(f)}{\pi^{2 s+2}(2 s+1) !} \delta_{s, t-\ell, j}(\ell) \\
- & \frac{1}{\gamma_{p}(\tau)} \sum_{j=0}^{r} d_{j} \sum_{t=2 q}^{2 q+[(p+1) / 2]-1} \frac{1}{N^{t+2}} \\
& \cdot \sum_{r=q}^{[t / 2]} \frac{A_{2 s+1}(f)}{\pi^{2 s+2}(2 s+1) !} \delta_{s, t, j}(1) \\
+ & o\left(N^{-2 q-[(p+1) / 2]-1}\right),
\end{aligned}
$$

which completes the proof in view of (B.2) and (B.3) (see Appendix B).

By repeating the observations of previous section, it is possible to deduce that, for getting the maximal convergence rate for odd values of $p$, the polynomial $Q_{r}(k)$ can be at most degree 0 polynomial, $Q_{r}(k)=Q_{0}(k) \equiv 1$. For even values of $p, Q_{r}(k)=Q_{1}(k)=1+d_{1} k$. In the first case, parameters $\tau_{k}$ are the roots of $L_{p}^{(2 q)}(x)$. In the second case, if $d_{1}=0$, we get the roots of $L_{p}^{(2 q)}(x)$ and if $d_{1}=-1 /(2 q+p)$, we get the roots of $L_{p}^{(2 q-1)}(x)$. The next two theorems immediately follow from Lemma 13 and identity (B.12) and, we omit the proofs.

Theorem 14. Let parameter $p \geq 1$ be odd, $f \in$ $C^{2 q+p+(p+1) / 2+2}[-1,1], q \geq 0, f^{(2 q+p+(p+1) / 2+2)} \in B V[-1,1]$, and

$$
f^{(2 k+1)}( \pm 1)=0, \quad k=0, \ldots, q-1 .
$$

Let $\theta_{k}, k=1, \ldots, p$, be defined by (26), where $\tau_{k}, k=1, \ldots, p$, are the roots of the generalized Laguerre polynomial $L_{p}^{(2 q)}(x)$. Then, the following estimates hold as $N \rightarrow \infty$ :

$$
R_{N, p}^{c}(f, \theta, \pm 1)=\frac{1}{N^{2 q+(p+1) / 2+1}}
$$




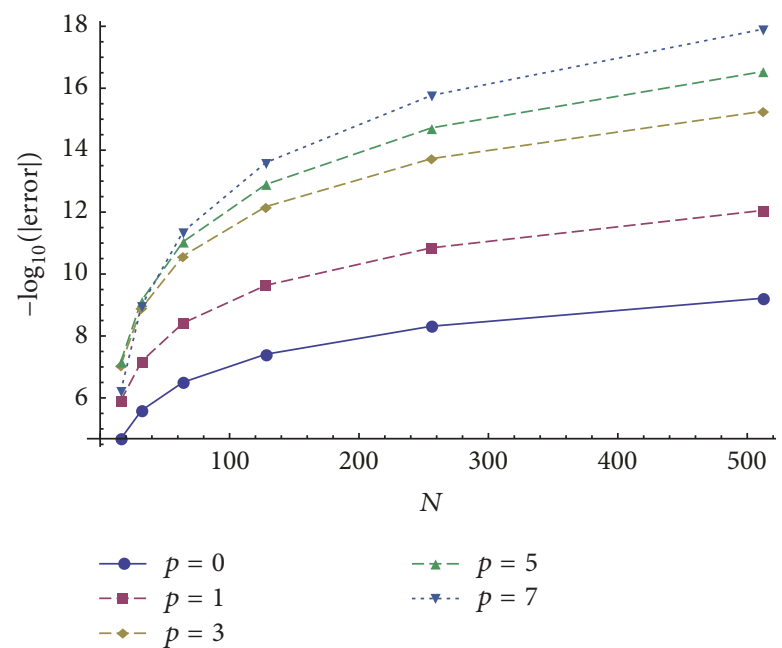

Figure 7: The values of $-\log _{10}\left(\max \left|R_{N, p}\left(f, \theta^{c}, \theta^{s}, \pm 1\right)\right|\right)$ while approximating (53) for different $N$ and $p$. Parameters $\tau_{k}^{c}$ and $\tau_{k}^{s}$, $k=1, \ldots, p$, are the roots of the generalized Laguerre polynomial $L_{p}^{(2 q)}(x)$.

$$
\begin{gathered}
\cdot \frac{A_{2 q+1}(f)}{\gamma_{p}(\tau) \pi^{2 q+2}(2 q+1) !}\left(\delta_{q, 2 q+(p+1) / 2,0}(0)\right. \\
\left.-\frac{1}{2} \delta_{q, 2 q+(p-1) / 2,0}(1)\right)+o\left(N^{-2 q-(p+1) / 2-1}\right), \\
R_{N, p}^{s}(f, \theta, \pm 1)= \pm \frac{1}{N^{2 q+(p+1) / 2+1}} \\
\cdot \frac{B_{2 q+1}(f)}{\gamma_{p}(\tau) \pi^{2 q+2}(2 q+1) !}\left(\widetilde{\delta}_{q, 2 q+(p+1) / 2,0}(0)\right. \\
\left.-\frac{1}{2} \widetilde{\delta}_{q, 2 q+(p-1) / 2,0}(1)\right)+o\left(N^{-2 q-(p+1) / 2-1}\right),
\end{gathered}
$$

\section{where $\delta$ and $\widetilde{\delta}$ are defined in Lemma 13.}

Figure 7 shows the result of approximation of (53) by the rational approximations with optimal values of parameters $\tau_{k}$, $k=1, \ldots, p$, as in Theorem 14 . We see that, by increasing $p$ ( $p$ is odd), we increase the accuracy of approximations at the points $x= \pm 1$. Note that $p=0$ corresponds to the classical expansion by the modified basis and we see that, in contrary to Figure 6, the optimal choice of parameters do have big positive impact on the accuracy.

Comparison of Theorems 9 and 14 reveals the problem of the optimal rational approximations which is in the difference of optimal values of parameters $\tau_{k}$ for $|x|<1$ and $x= \pm 1$. On $|x|<1$ and $x= \pm 1$, the optimal values are the roots of $L_{p}^{(2 q+1)}(x)$ and $L_{p}^{(2 q)}(x)$, respectively. The choice of $L_{p}^{(2 q)}(x)$ will result in better accuracy on overall $[-1,1]$ by the uniform norm, but on $|x|<1$ the rate of convergence will be worse by factor $O(N)$.

Next theorem explores even values of $p$.
Theorem 15. Let parameter $p \geq 2$ be even, $f \in$ $C^{2 q+p+p / 2+2}[-1,1], q \geq 0, f^{(2 q+p+p / 2+2)} \in B V[-1,1]$, and

$$
f^{(2 k+1)}( \pm 1)=0, \quad k=0, \ldots, q-1 .
$$

Assume the polynomial

$$
\sum_{k=0}^{p}\left(\begin{array}{l}
p \\
k
\end{array}\right) \frac{1+d_{1}(p-k)}{(2 q+k) !}(-1)^{k} x^{k}
$$

has only real-valued and nonzero roots $x=z_{k}, k=1, \ldots, p$, and let $\theta_{k}$ be defined by (26) with $\tau_{k}=z_{k}$. Then, the following asymptotic expansions hold:

$$
\begin{aligned}
R_{N, p}^{c}(f, \theta, \pm 1) & \\
= & \frac{1}{N^{2 q+p / 2+1}} \frac{A_{2 q+1}(f)}{\gamma_{p}(\tau) \pi^{2 q+2}(2 q+1) !} \Phi_{q, p}\left(d_{1}\right) \\
& \quad+o\left(N^{-2 q-p / 2-1}\right), \quad N \longrightarrow \infty, \\
R_{N, p}^{s}(f, \theta, \pm 1) & \\
= & \pm \frac{1}{N^{2 q+[(p+1) / 2]+1}} \frac{B_{2 q+1}(f)}{\gamma_{p}(\tau) \pi^{2 q+2}(2 q+1) !} \widetilde{\Phi}_{q, p}\left(d_{1}\right) \\
& +o\left(N^{-2 q-p / 2-1}\right), \quad N \longrightarrow \infty,
\end{aligned}
$$

where

$$
\begin{aligned}
\Phi_{q, p} & \left(d_{1}\right) \\
= & \delta_{q, 2 q+p / 2,0}(0)-\delta_{q, 2 q+p / 2-1,0}(1) \\
& \quad+d_{1}\left(\delta_{q, 2 q+p / 2,1}(0)-\frac{1}{2} \delta_{q, 2 q+p / 2-1,1}(1)\right), \\
\widetilde{\Phi}_{q, p}\left(d_{1}\right) & \widetilde{\delta}_{q, 2 q+p / 2,0}(0)-\widetilde{\delta}_{q, 2 q+p / 2-1,0}(1) \\
& +d_{1}\left(\widetilde{\delta}_{q, 2 q+p / 2,1}(0)-\frac{1}{2} \widetilde{\delta}_{q, 2 q+p / 2-1,1}(1)\right),
\end{aligned}
$$

with $\delta$ and $\tilde{\delta}$ defined in Lemma 13.

Estimates (113) and (114) are valid if polynomial (112) has only real-valued and nonzero roots. As we mentioned above, in two particular cases when $d_{1}=0$ and $d_{1}=-1 /(2 q+p)$, the roots of the polynomial coincide with the roots of $L_{p}^{(2 q)}(x)$ and $L_{p}^{(2 q-1)}(x)$, respectively. Both Laguerre polynomials have only real-valued and positive roots and Theorem 15 is valid in both cases. The choice of polynomial $L_{p}^{(2 q)}(x)$ is reasonable as it will provide optimal rational approximation both on $|x|<1$ (see Theorem 10) and at $x= \pm 1$ for some $p$ and $q$.

Figure 8 shows the result of application of the rational approximations to function (53) with optimal values of parameters $\tau_{k}, k=1, \ldots, p$, as the roots of $L_{p}^{(2 q)}(x)$.

Now, we show that, for some $p$ and $q$, estimates (113) and (114) can be improved with appropriate selection of parameter 


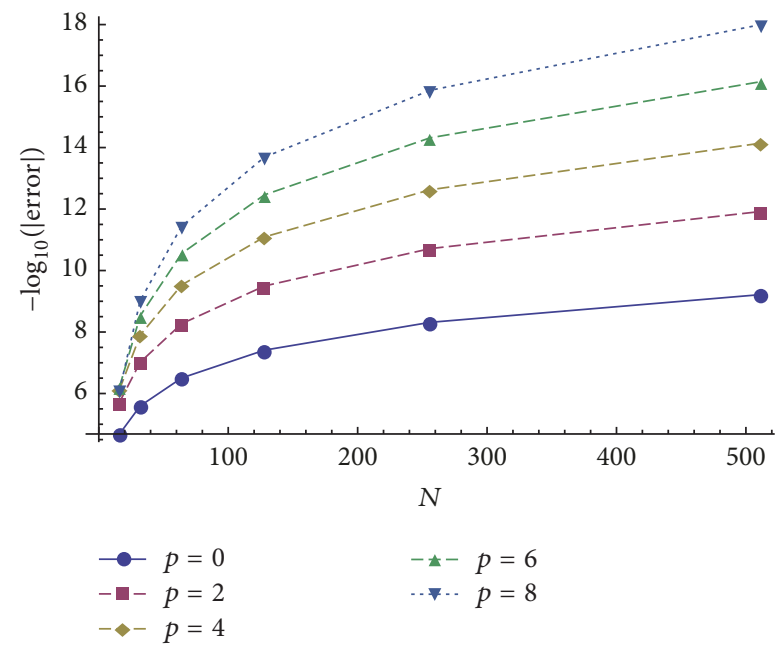

Figure 8: The values of $-\log _{10}\left(\max \left|R_{N, p}\left(f, \theta^{c}, \theta^{s}, \pm 1\right)\right|\right)$ while approximating (53) for different $N$ and $p$. Parameters $\tau_{k}^{c}$ and $\tau_{k}^{s}$, $k=1, \ldots, p$, are the roots of the generalized Laguerre polynomial $L_{p}^{(2 q)}(x)$.

$d_{1}$. Assume that, for given $p$ and $q$, it is possible to vanish $\Phi_{q, p}\left(d_{1}\right)$ by appropriate selection of $d_{1}$ in (113). Then, we derive

$$
R_{N, p}^{c}\left(f, \theta^{c}, \theta^{s}, \pm 1\right)=o\left(N^{-2 q-p / 2-1}\right),
$$

or

$$
R_{N, p}^{c}\left(f, \theta^{c}, \theta^{s}, \pm 1\right)=O\left(N^{-2 q-p / 2-2}\right)
$$

in case of smoother functions. Similarly, if $\widetilde{\Phi}_{q, p}\left(d_{1}\right)=0$ by appropriate selection of parameter $d_{1}$, then,

$$
R_{N, p}^{s}\left(f, \theta^{c}, \theta^{s}, \pm 1\right)=o\left(N^{-2 q-p / 2-1}\right) \text {, }
$$

or

$$
R_{N, p}^{s}\left(f, \theta^{c}, \theta^{s}, \pm 1\right)=O\left(N^{-2 q-p / 2-2}\right)
$$

in case of smoother functions.

The problem is that we cannot vanish both $\Phi_{q, p}\left(d_{1}\right)$ and $\widetilde{\Phi}_{q, p}\left(d_{1}\right)$ simultaneously by the same $d_{1}$. Hence, we decompose a function into even and odd parts and perform separate optimizations in terms of parameter $d_{1}$. In order to choose parameter $d_{1}$ appropriately, we need to have

$$
\begin{aligned}
& \delta_{q, 2 q+p / 2,1}(0)-\frac{1}{2} \delta_{q, 2 q+p / 2-1,1}(1) \neq 0, \\
& \widetilde{\delta}_{q, 2 q+p / 2,1}(0)-\frac{1}{2} \widetilde{\delta}_{q, 2 q+p / 2-1,1}(1) \neq 0
\end{aligned}
$$

for even and odd functions, respectively.

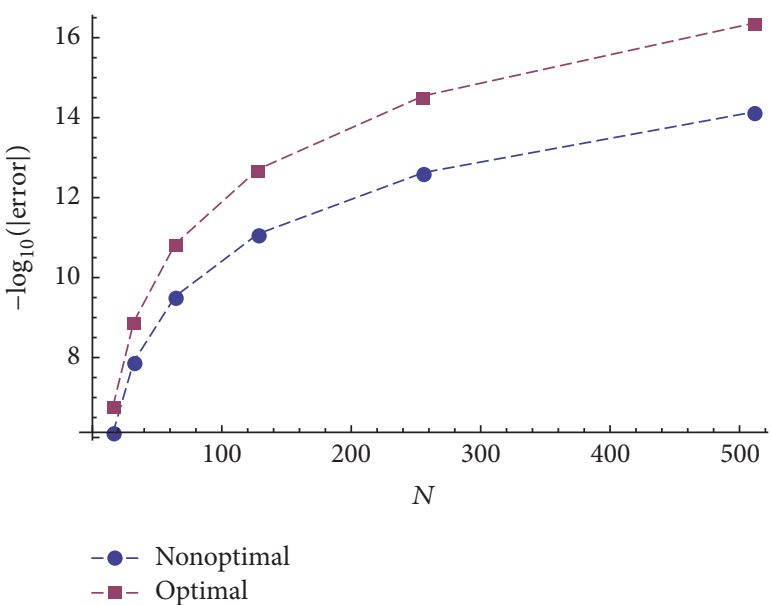

Figure 9: The values of $-\log _{10}\left(\max \left|R_{N, p}\left(f, \theta^{c}, \theta^{s}, \pm 1\right)\right|\right)$ for $p=4$ and different $N$ while approximating (53). In "nonoptimal" case, parameters $\tau_{k}^{c}$ and $\tau_{k}^{s}, k=1, \ldots, p$, are the roots of $L_{p}^{(2 q)}(x)$. In "optimal" case, the parameters are chosen from Tables 1 and 2 for $p=4$ and $q=1$.

Then, we put

$$
\begin{aligned}
d_{1} & =d_{p}^{\text {even }}(q) \\
& =\frac{-\delta_{q, 2 q+p / 2,0}(0)+\delta_{q, 2 q+p / 2-1,0}(1)}{\delta_{q, 2 q+p / 2,1}(0)-(1 / 2) \delta_{q, 2 q+p / 2-1,1}(1)}, \\
d_{1} & =d_{p}^{\text {odd }}(q) \\
& =\frac{-\widetilde{\delta}_{q, 2 q+p / 2,0}(0)+\widetilde{\delta}_{q, 2 q+p / 2-1,0}(1)}{\widetilde{\delta}_{q, 2 q+p / 2,1}(0)-(1 / 2) \widetilde{\delta}_{q, 2 q+p / 2-1,1}(1)} .
\end{aligned}
$$

Finally, we put $d_{p}^{\text {even }}(q)$ and $d_{p}^{\text {odd }}(q)$ into (112) and if that polynomials have only real-valued and nonzero roots, the optimization process will succeed. Tables 1 and 2 show that, except some special cases, we can optimize estimates of Theorem 15.

Figures 9 and 10 show the errors at $x= \pm 1$ while approximating (53) with rational approximations, where parameters $\tau_{k}^{c}$ and $\tau_{k}^{s}, k=1, \ldots, p$, are selected according to Tables 1 and 2 for even and odd parts of the function, respectively. We called this approach "optimal" in the figures. For comparison, we showed also the result of approximations with parameters $\tau_{k}^{c}$ and $\tau_{k}^{s}, k=1, \ldots, p$, as the roots of $L_{p}^{(2 q)}(x)$ (see Figure 8 and Theorem 15). We called the latest "nonoptimal" in the figures. We see the impact of optimizations on the accuracy of the rational approximations at $x= \pm 1$.

Throughout the paper, we systematically required that approximated function $f$ obeys first derivative conditions (8). Without those conditions, the convergence rate will remain slow. This is due to function jumps in certain derivatives at the endpoints $x= \pm 1$. If these jumps are known, the convergence acceleration can be achieved by well-known polynomial subtraction approach. For the classical Fourier series this approach has a very long history (see [3, 22-28]). 
TABLE 1: The values of $d_{p}^{\text {even }}(q)$ and the roots of (112) for $p=2,4,6$ and $0 \leq q \leq 6$.

\begin{tabular}{|c|c|c|c|c|c|c|c|}
\hline$q$ & 0 & 1 & 2 & 3 & 4 & 5 & 6 \\
\hline$d_{2}^{\text {even }}(q)$ & -1 & 1 & $1 / 3$ & $1 / 5$ & $1 / 7$ & $1 / 9$ & $1 / 11$ \\
\hline$\tau_{1}$ & -1.41 & 2.71 & 4.26 & 5.89 & 7.57 & 9.28 & 11.01 \\
\hline$\tau_{2}$ & 1.41 & 13.29 & 11.74 & 13.31 & 15.29 & 17.39 & 19.53 \\
\hline$d_{4}^{\text {even }}(q)$ & $-3 / 14$ & $-3 / 2$ & $3 / 10$ & $3 / 22$ & $3 / 34$ & $3 / 46$ & $3 / 58$ \\
\hline$\tau_{1}$ & 0.12 & -27.44 & 2.61 & 3.81 & 5.10 & 6.45 & 7.85 \\
\hline$\tau_{2}$ & 1.09 & 1.57 & 5.86 & 7.50 & 9.23 & 10.99 & 12.78 \\
\hline$\tau_{3}$ & 3.46 & 4.44 & 10.85 & 12.72 & 14.76 & 16.86 & 18.98 \\
\hline$\tau_{4}$ & 7.91 & 9.43 & 22.28 & 21.43 & 23.15 & 25.36 & 27.71 \\
\hline$d_{6}^{\text {even }}(q)$ & $-1 / 11$ & $-1 / 5$ & 1 & $1 / 7$ & $1 / 13$ & $1 / 19$ & $1 / 25$ \\
\hline$\tau_{1}$ & 0.18 & -0.74 & 1.96 & 2.90 & 3.95 & 5.07 & 6.25 \\
\hline$\tau_{2}$ & 1.00 & 1.34 & 4.28 & 5.57 & 6.98 & 8.46 & 9.98 \\
\hline$\tau_{3}$ & 2.60 & 3.41 & 7.51 & 9.04 & 10.76 & 12.54 & 14.35 \\
\hline$\tau_{4}$ & 5.15 & 6.45 & 11.99 & 13.62 & 15.55 & 17.59 & 19.67 \\
\hline$\tau_{5}$ & 8.96 & 10.74 & 18.54 & 19.91 & 21.87 & 24.08 & 26.38 \\
\hline$\tau_{6}$ & 14.84 & 17.18 & 75.72 & 31.25 & 31.35 & 33.32 & 35.69 \\
\hline
\end{tabular}

TABLE 2: The values of $d_{p}^{\text {odd }}(q)$ and the roots of (112) for $p=2,4,6$ and $0 \leq q \leq 4$.

\begin{tabular}{lccccccc}
\hline$q$ & 0 & 1 & 2 & 3 & 4 & 5 \\
\hline$d_{2}^{\text {odd }}(q)$ & - & - & $1 / 2$ & $1 / 4$ & $1 / 6$ & $1 / 8$ & $1 / 10$ \\
$\tau_{1}$ & - & - & 4.417 & 6 & 7.653 & 9.347 \\
$\tau_{2}$ & - & - & 13.58 & 14 & 15.68 & 17.65 \\
\hline$d_{4}^{\text {odd }}(q)$ & $-3 / 20$ & $-3 / 8$ & $3 / 4$ & $3 / 16$ & $3 / 28$ & $3 / 40$ & 19.73 \\
$\tau_{1}$ & 0.23 & -1.95 & 2.70 & 3.87 & 5.14 & 6.48 \\
$\tau_{2}$ & 1.35 & 1.83 & 6.09 & 7.63 & 9.31 & 11.05 \\
$\tau_{3}$ & 3.77 & 4.96 & 11.41 & 13.01 & 14.93 & 16.98 \\
$\tau_{4}^{\text {odd }}$ & 8.249 & 10.16 & 35.80 & 22.99 & 23.76 & 25.69 \\
$d_{6}(q)$ & $-1 / 14$ & $-1 / 8$ & $-1 / 2$ & $1 / 4$ & $1 / 10$ & $1 / 16$ \\
$\tau_{1}$ & 0.20 & 0.53 & -16.59 & 2.94 & 3.98 & 27.83 \\
$\tau_{2}$ & 1.06 & 1.80 & 2.07 & 5.67 & 7.04 & 5.09 \\
$\tau_{3}$ & 2.71 & 3.88 & 4.52 & 9.23 & 10.86 & 8.50 \\
$\tau_{4}$ & 5.29 & 6.92 & 7.93 & 13.95 & 15.73 & 12.60 \\
$\tau_{5}$ & 9.14 & 11.23 & 12.62 & 20.58 & 22.21 & 17.70 \\
$\tau_{6}$ & 15.04 & 17.65 & 19.45 & 37.63 & 32.58 & 10.07 \\
\hline
\end{tabular}

For modified expansions this approach is explored in $[3,5,7]$. More specifically, we write $f$ (see [3]) in the terms of its Lanczos representation:

$$
f=\left(f-g_{k}\right)+g_{k},
$$

where functions (polynomials) $g_{k}$ are chosen as such to satisfy the conditions:

$$
f^{(2 r+1)}( \pm 1)=g_{k}^{(2 r+1)}( \pm 1), \quad r=0, \ldots, k-1 .
$$

Since $f-g_{k}$ obeys the first $k$ derivative conditions, the new approximation

$$
M_{N}^{k}(f, x)=M_{N}\left(f-g_{k}, x\right)+g_{k}
$$

will converge with the same rate as if $f$ obeyed those conditions. This is the polynomial subtraction technique known also as Krylov-Lanczos approach. If the jumps of $f$ are unknown, their values can be approximated by solution of the corresponding system of linear equations (see [22]).

The same approach can be applied also for the MTRapproximations and in that case all theorems of this paper will remain valid without the requirements of the derivatives at the endpoints $x= \pm 1$.

\section{Conclusion}

In this paper, we investigated the convergence of the MTRapproximations $M_{N, p}$ with parameters $\theta^{c}$ and $\theta^{s}$ defined by (24). The main goal of the paper was to show that by appropriate selection of parameters $\tau^{c}$ and $\tau^{s}$ it was possible to improve substantially the pointwise convergence of the approximations. We accomplished the main goal by calculating the exact constants of the main terms of 


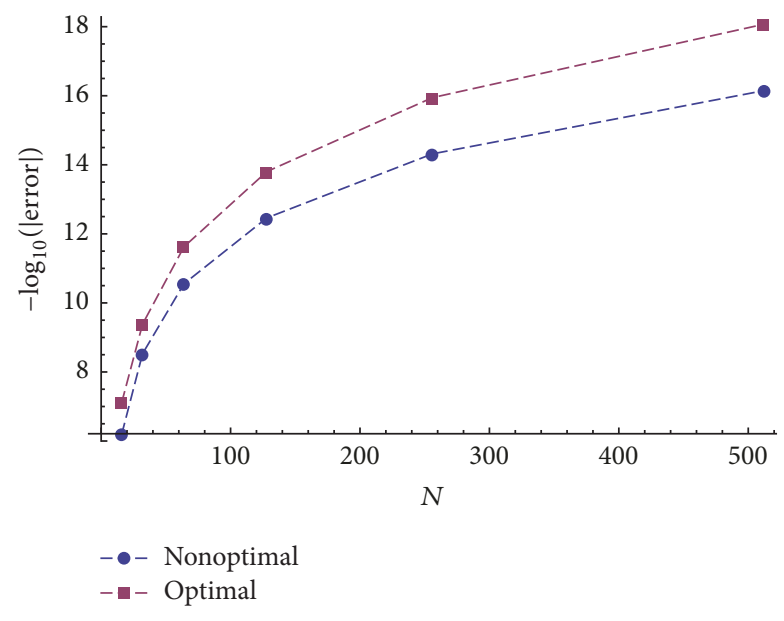

Figure 10: The values of $-\log _{10}\left(\max \left|R_{N, p}\left(f, \theta^{c}, \theta^{s}, \pm 1\right)\right|\right)$ for $p=6$ and different $N$ while approximating (53). In "nonoptimal" case, parameters $\tau_{k}^{c}$ and $\tau_{k}^{s}, k=1, \ldots, p$, are the roots of $L_{p}^{(2 q)}(x)$. In "optimal" case, the parameters are chosen from Tables 1 and 2 for $p=6$ and $q=1$.

asymptotic errors and by eliminating those constants through appropriate selection of the approximation parameters. We showed that the optimization depends whether $|x|<1$ or $x= \pm 1$ and also whether parameter $p$ is odd or even.

Theorem 6 provides general estimate on $|x|<1$ proving that, without optimal selection of parameters $\tau_{k}^{c}$ and $\tau_{k}^{s}$, $k=1, \ldots, p$, the rational approximations have convergence rate $O\left(N^{-2 q-p-2}\right)$ as $N \rightarrow \infty$ if an approximated function has enough smoothness and obeys the first $q$ derivative conditions (see (8)). Compared with the modified Fourier expansions (see Theorems 1 and 2 or the same theorem with $p=0)$, the improvement is by factor $O\left(N^{p}\right)$ as $N \rightarrow \infty$.

Theorem 9 gave the optimal choice for parameters $\tau_{k}$ when $|x|<1$ and $p$ was odd. If $\tau_{k}^{c}=\tau_{k}^{s}, k=1, \ldots, p$, were the roots of the generalized Laguerre polynomial $L_{p}^{(2 q+1)}(x)$ then the rational approximations had convergence rate $O\left(N^{-2 q-p-[(p+1) / 2]-2}\right)$ with improvement by factor $O\left(N^{[(p+1) / 2]}\right)$ compared to nonoptimal choice of parameters (Theorem 6). The improvement was by factor $O\left(N^{[(p+1) / 2]+p}\right)$ compared to the expansions by the modified Fourier basis.

In case of even values of $p$ and $|x|<1$ (Theorem 10), possible selection set of optimal parameters is wider. If for given $p$ and $q$ the polynomial

$$
\sum_{k=0}^{p}\left(\begin{array}{l}
p \\
k
\end{array}\right) \frac{1+c_{1}(p-k)}{(2 q+1+k) !}(-1)^{k} x^{k}
$$

has only nonzero and real-valued roots $x=z_{k}, k=1, \ldots, p$, then, selection $\tau_{k}^{s}=\tau_{k}^{c}=z_{k}$ provides better convergence rate $O\left(N^{-2 q-p-[p / 2]-2}\right)$ compared to the estimate of Theorem 6 and improvement is by factor $O\left(N^{[p / 2]}\right)$. Improvement is by factor $O\left(N^{[p / 2]+p}\right)$ compared to the expansions by the modified Fourier basis. The problem is to find the values of $c_{1}$ in (126) for which it will have only real-valued and nonzero roots. In two cases it is obvious. When $c_{1}=0$, the roots of (126) coincide with the roots of $L_{p}^{(2 q+1)}(x)$. When $c_{1}=-1 /(2 q+p+$ $1)$, the roots coincide with the ones of $L_{p}^{(2 q)}(x)$. In both cases all roots are positive.

Theorem 11 imparts the convergence rate $O\left(N^{-2 q-1}\right)$ of the rational approximations at $x= \pm 1$ without optimal selection of parameters. Comparison with Theorems 1 and 2 shows no improvement. Moreover, as our experiments show (see Figure 6) rational approximations without reasonable selection of parameters $\tau_{k}$ can perform worse at $x=$ \pm 1 compared to the expansions by the modified Fourier basis.

Theorem 14 found the optimal values of parameters for odd $p$ for better convergence rate at $x= \pm 1$. It proved that the best accuracy could be achieved when parameters $\tau_{k}^{s}=\tau_{k}^{c}$ were the roots of the generalized Laguerre polynomial $L_{p}^{(2 q)}(x)$. For that choice, the convergence rate was $O\left(N^{-2 q-[(p+1) / 2]-1}\right)$ and improvement was by factor $O\left(N^{[(p+1) / 2]}\right)$ compared to the modified Fourier expansions.

We see that when $p$ is odd, the optimal choices for $|x|<1$ and $x= \pm 1$ are different. The choice of polynomial $L_{p}^{(2 q)}(x)$ will provide the minimal uniform error on $[-1,1]$, but, for $|x|<1$, the convergence rate will be worse by factor $O(N)$ compared to the optimal choice $L_{p}^{(2 q+1)}(x)$.

In case of even $p$, we obtained similar results. Theorem 15 outlines the set of optimal parameters. If for given $p$ and $q$ the polynomial

$$
\sum_{k=0}^{p}\left(\begin{array}{l}
p \\
k
\end{array}\right) \frac{1+d_{1}(p-k)}{(2 q+k) !}(-1)^{k} x^{k}
$$

has only real-valued and nonzero roots $x=z_{k}$ for some $d_{1}$, then, selection $\tau_{k}^{c}=\tau_{k}^{s}=z_{k}$ will provide convergence rate $O\left(N^{-2 q-[p / 2]-1}\right)$ with improvement by factor $O\left(N^{[p / 2]}\right)$ compared to the modified Fourier expansions. The problem is the same as that for polynomial (126). Polynomial (127) must have only real-valued and nonzero roots for the selected $d_{1}$. Fortunately, two such selections are known. When $d_{1}=0$ or $d_{1}=-1 /(2 q+p)$, the roots of (127) coincide with the ones of $L_{p}^{(2 q)}(x)$ and $L_{p}^{(2 q-1)}(x)$, respectively. The choice of $L_{p}^{(2 q)}(x)$ is better as it will provide optimal approximations for both $|x|<1$ and $x= \pm 1$. However, estimates of Theorem 15 allow determining parameter $d_{1}$ for even more better convergence rate. Tables 1 and 2 show some values of $d_{1}$ and parameters $\tau_{k}$ that will provide convergence rate $O\left(N^{-2 q-[p / 2]-2}\right)$ with improvement by factor $O(N)$. The problem is that the latest choice is not optimal for $|x|<$ 1. It will give worse accuracy compared to the optimal selection for $|x|<1$. Convergence rate will degrade by factor $O(N)$. As in case of odd $p$, a user of the algorithms must decide which choice will be more appropriate to select, the best approximation on overall $[-1,1]$ with worse accuracy on $|x|<1$, or the best accuracy on the latest one. 


\section{Appendix}

\section{A. Laguerre Polynomials}

The differential equation [29]

$$
x y^{\prime \prime}+(1-x) y^{\prime}+p y=0, \quad x \geq 0
$$

is known as Laguerre differential equation. If $p$ is a natural number, the solution of (A.1) reduces to a polynomial. When properly normalized, the polynomial is known as the Laguerre polynomial $L_{p}(x)$. One of the normalizations is given by the Rodrigues formula:

$$
L_{p}(x)=\frac{e^{x}}{p !} \frac{d^{p}}{d x^{p}}\left(e^{-x} x^{p}\right) .
$$

This choice leads to the following closed form for the Laguerre polynomials:

$$
L_{p}(x)=\sum_{k=0}^{p}\left(\begin{array}{l}
p \\
k
\end{array}\right) \frac{(-1)^{k}}{k !} x^{k} .
$$

Generalized Laguerre polynomials $L_{p}^{(\alpha)}(x)$ are defined by the following relation:

$$
L_{p}^{(\alpha)}(x)=(-1)^{\alpha} \frac{d^{\alpha}}{d x^{\alpha}}\left[L_{p+\alpha}(x)\right]
$$

with the closed form

$$
L_{p}^{(\alpha)}(x)=\sum_{k=0}^{p}(-1)^{k} \frac{(p+\alpha) !}{k !(p-k) !(\alpha+k) !} x^{k} .
$$

The generalized Laguerre polynomials are orthogonal over $[0, \infty)$ with the weight function $x^{\alpha} e^{-x}$

$$
\int_{0}^{\infty} x^{\alpha} e^{-x} L_{n}^{(\alpha)}(x) L_{m}^{(\alpha)}(x) d x=\frac{\Gamma(n+\alpha+1)}{n !} \delta_{n, m}
$$

which leads to the property that $L_{p}^{(\alpha)}(x)$ has $p$ real-valued and strictly positive simple roots.

\section{B. Some Combinatorial Identities}

In Section 2, we defined $\delta_{s, t}(w)$ by the following equation (see (104)):

$$
\begin{aligned}
& \delta_{s, t, j}(w) \\
& =(2 q+p) ! \sum_{k=0}^{p}\left(\begin{array}{l}
p \\
k
\end{array}\right) \beta_{k, s, t}(0) \frac{(p-k+t+w) !}{(2 q+p-k) !} k^{j},
\end{aligned}
$$

where $\beta_{k, s, t}$ are defined by (35). We explore some properties of $\delta_{s, t, j}(w)$.

First, we prove that

$$
\delta_{s, t, j}(w)=0
$$

when

$$
\begin{aligned}
2 q & \leq t \leq 2 q+\left[\frac{p+1-w-j}{2}\right]-1, \\
q & \leq s \leq\left[\frac{t}{2}\right] .
\end{aligned}
$$

We have

$$
\delta_{s, t, j}(w)=(2 q+p) ! \sum_{k=0}^{p}\left(\begin{array}{l}
p \\
k
\end{array}\right) \frac{(p-k+t+w) !}{(2 q+p-k) !} \sum_{\ell=0}^{t-2 s} \frac{k^{t+j-2 s-\ell}}{(t-2 s-\ell) !(p-k+\ell) !} \sum_{u=0}^{p-k}(-1)^{u} u^{p-k+\ell}\left(\begin{array}{c}
p-k \\
u
\end{array}\right) .
$$

Then,

$$
\delta_{s, t, j}(w)=(2 q+p) !(-1)^{p} \sum_{k=0}^{p}(-1)^{k}\left(\begin{array}{l}
p \\
k
\end{array}\right) \frac{(p-k+t+w) !}{(2 q+p-k) !} \sum_{u=0}^{t-2 s} k^{t+j-2 s-u} \frac{(p-k) !}{(t-2 s-u) !(p-k+u) !} S(p-k+u, p-k)
$$

where $S(n, k)$ are the Stirling numbers of the second kind (see [30]). Applying the well-known property [30] of the Stirling numbers

$$
S(k+m, k)=\sum_{t=0}^{m}\left(\begin{array}{c}
k+m \\
m+t
\end{array}\right) c_{t}(m), \quad m \geq 0
$$

where $c_{t}(m)$ are the associated Stirling numbers of the second kind, we can write

$$
S(p-k+u, p-k)=\sum_{r=0}^{u}\left(\begin{array}{c}
p-k+u \\
u+r
\end{array}\right) c_{r}(u) .
$$

Thus, for $\delta_{s, t, j}(w)$, we obtain

$$
\delta_{s, t, j}(w)=(2 q+p) !(-1)^{p} \sum_{u=0}^{t-2 s} \frac{1}{(t-2 s-u) !} \sum_{r=0}^{u} \frac{c_{r}(u)}{(u+r) !} \sum_{k=0}^{p} k^{t+j-2 s-u}(-1)^{k}\left(\begin{array}{l}
p \\
k
\end{array}\right) \frac{(p-k+t+w) !(p-k) !}{(2 q+p-k) !(p-k-r) !}
$$


It remains to notice that the expression

$$
k^{t+j-2 s-u} \frac{(p-k+t+w) !(p-k) !}{(2 q+p-k) !(p-k-r) !}
$$

is a $(2 t-2 s-u+r-2 q+j+w)$-degree polynomial of $k$, and hence we can write

$$
\begin{gathered}
k^{t-2 s-u+j} \frac{(p-k+t+w) !(p-k) !}{(2 q+p-k) !(p-k-r) !} \\
=\sum_{m=0}^{2 t+j+w-2 s-u+r-2 q} d_{m} k^{m}
\end{gathered}
$$

with some coefficients $d_{m}$. Therefore,

$$
\delta_{s, t, j}(w)=(2 q+p) !(-1)^{p} \sum_{u=0}^{t-2 s} \frac{1}{(t-2 s-u) !} \sum_{r=0}^{u} \frac{c_{r}(u)}{(u+r) !} \sum_{m=0}^{2 t+j+w-2 s-u+r-2 q} d_{m} \alpha_{m, p},
$$

where $\alpha_{m, p}$ are defined by (37). It is easy to verify that $2 t+j+$ $w-2 s-u+r-2 q<p$, which completes the proof as $\alpha_{m, p}=0$ for $m<p$.

Second, in view of (B.11), we similarly prove that

$$
\begin{aligned}
\delta_{s, t, j}(w)= & 0, \\
& t=2 q+\left[\frac{p+1-j-w}{2}\right], q<s \leq\left[\frac{t}{2}\right] .
\end{aligned}
$$

In Section 3, we defined coefficients $\sigma_{s, t, j}(w)$ as follows:

$$
\begin{aligned}
\sigma_{s, t, j}(w) & =(2 q+p \\
+1) ! & \sum_{k=0}^{p}\left(\begin{array}{l}
p \\
k
\end{array}\right) \beta_{k, s, t}(w) \frac{(p-k+t+1) !}{(2 q+p+1-k) !} k^{j},
\end{aligned}
$$

where $\beta_{k, s, t}$ are defined in Lemma 5. Similar to (B.11), we derive that

$$
\sigma_{s, t, j}(w)=(2 q+p+1) !(-1)^{p+w} \sum_{u=0}^{t-2 s-w} \frac{1}{(t-2 s-w-u) !} \sum_{r=0}^{u} \frac{c_{r}(u)}{(u+r) !} \sum_{m=0}^{2 t-2 s-u-w+r-2 q+j} d_{m} \alpha_{m, p},
$$

and, here, we observe that

$$
\begin{aligned}
& \sigma_{s, t, j}(w)=0 \\
& 2 q \leq t \leq 2 q+\left[\frac{w+p-j+1}{2}\right]-1, q \leq s \leq\left[\frac{t-w}{2}\right], \\
& \sigma_{s, t, j}(w)=0 \\
& t=2 q+\left[\frac{w+p-j+1}{2}\right], q<s \leq\left[\frac{t-w}{2}\right] .
\end{aligned}
$$

\section{Conflicts of Interest}

The authors declare that there are no conflicts of interest regarding the publication of this paper.

\section{Acknowledgments}

The research of the second author was supported by SCS Grant 16A-1a40.

\section{References}

[1] M. G. Krein, "On a special class of differential operators," Doklady AN USSR, vol. 2, pp. 345-349, 1935.

[2] B. Adcock, "Univariate modified Fourier methods for second order boundary value problems," BIT Numerical Mathematics, vol. 49, no. 2, pp. 249-280, 2009.
[3] B. Adcock, Modified Fourier Expansions: Theory, Construction and Applications [Ph.D. thesis], Trinity Hall, University of Cambridge, England, UK, July 2010.

[4] B. Adcock, "Multivariate modified Fourier series and application to boundary value problems," Numerische Mathematik, vol. 115 , no. 4, pp. 511-552, 2010.

[5] B. Adcock, "Convergence acceleration of modified Fourier series in one or more dimensions," Mathematics of Computation, vol. 80, no. 273, pp. 225-261, 2011.

[6] T. K. Bakaryan, "On a convergence of the modified FourierPade approximations," Armenian Journal of Mathematics, vol. 8, no. 2, pp. 120-144, 2016.

[7] D. Huybrechs, A. Iserles, S. Nørsett, and P. From high, "From high oscillation to rapid approximation IV: accelerating convergence," IMA Journal of Numerical Analysis, vol. 31, pp. 442-468, 2011.

[8] A. Iserles, S. Nørsett, and P. From high, "oscillation to rapid approximation. I. Modified Fourier expansions," IMA Journal of Numerical Analysis, vol. 28, no. 4, pp. 862-887, 2008.

[9] A. Iserles and S. P. Nørsett, "From high oscillation to rapid approximation. III. Multivariate expansions," IMA Journal of Numerical Analysis, vol. 29, no. 4, pp. 882-916, 2009.

[10] S. Olver, "On the convergence rate of a modified Fourier series," Mathematics of Computation, vol. 78, no. 267, pp. 1629-1645, 2009.

[11] A. Zygmund, Trigonometric Series, vol. 1,2, Cambridge University Press, Cambridge, UK, 1959. 
[12] G. A. Baker Jr. and P. Graves-Morris, "Padé approximants," in Encyclopedia of Mathematics and its Applications, Cambridge University Press, Cambridge, UK, 2nd edition, 1996.

[13] J. F. Geer, "Rational trigonometric approximations using Fourier series partial sums," Journal of Scientific Computing, vol. 10, no. 3, pp. 325-356, 1995.

[14] A. Nersessian and A. Poghosyan, "On a rational linear approximation of Fourier series for smooth functions," Journal of Scientific Computing, vol. 26, no. 1, pp. 111-125, 2006.

[15] A. Poghosyan, "On a convergence of the Fourier-Pade approximation," Armenian Journal of Mathematics, vol. 4, no. 2, pp. 4979, 2012.

[16] A. Poghosyan, "On some optimizations of trigonometric interpolation using Fourier discrete coefficients," Armenian Journal of Mathematics, vol. 4, no. 2, pp. 80-97, 2012.

[17] A. Poghosyan, "On a convergence of the Fourier-Pade interpolation," Armenian Journal of Mathematics, vol. 5, no. 1, pp. 1-25, 2013.

[18] A. Poghosyan, "On a fast convergence of the rationaltrigonometric-polynomial interpolation," Advances in Numerical Analysis, Art. ID 315748, 13 pages, 2013.

[19] A. Poghosyan, "On a convergence of the rational-trigonometric-polynomial approximations realized by the roots of the Laguerre polynomials," Natsionalnaya Akademiya Nauk Armenii. Izvestiya. Matematika, vol. 48, no. 6, pp. 82-91, 2013.

[20] A. Poghosyan, "Asymptotic behavior of the Krylov-Lanczos interpolation," Analysis and Applications, vol. 7, no. 2, pp. 199211, 2009.

[21] V. Kac and P. Cheung, Quantum Calculus, Springer, New York, NY, USA, 2001.

[22] A. Barkhudaryan, R. Barkhudaryan, and A. Poghosyan, "Asymptotic behavior of Eckhoff's method for Fourier series convergence acceleration," Analysis in Theory and Applications, vol. 23, no. 3, pp. 228-242, 2007.

[23] K. S. Eckhoff, "Accurate and efficient reconstruction of discontinuous functions from truncated series expansions," Mathematics of Computation, vol. 61, no. 204, pp. 745-763, 1993.

[24] K. S. Eckhoff, "Accurate reconstructions of functions of finite regularity from truncated Fourier series expansions," Mathematics of Computation, vol. 64, no. 210, pp. 671-690, 1995.

[25] K. S. Eckhoff, "On a high order numerical method for functions with singularities," Mathematics of Computation, vol. 67, no. 223, pp. 1063-1087, 1998.

[26] A. Krylov, On approximate calculations. Lectures delivered in 1906, Tipolitography of Birkenfeld, St. Petersburg, Russia, 1907.

[27] C. Lanczos, Discourse on Fourier series, vol. 76 of Classics in Applied Mathematics, Society for Industrial and Applied Mathematics (SIAM), Philadelphia, PA, 2016.

[28] A. Poghosyan, "On an auto-correction phenomenon of the Krylov-Gottlieb-Eckhoff method," IMA Journal of Numerical Analysis (IMAJNA), vol. 31, no. 2, pp. 512-527, 2011.

[29] M. Abramowitz and I. A. Stegun, Handbook of Mathematical Functions with Formulas, Graphs, And Mathematical Tables, vol. 55 of National Bureau of Standards Applied Mathematics Series, Superintendent of Documents, U.S. Government Printing Office, Washington, DC, USA, 1964.

[30] J. Riordan, Combinatorial Identities, Wiley, New York, NY, USA, 1979. 


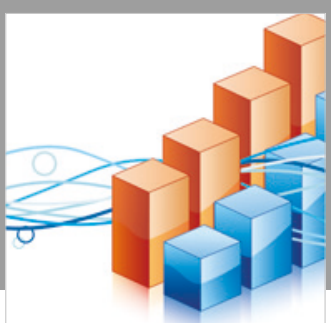

Advances in

Operations Research

\section{-n-m}
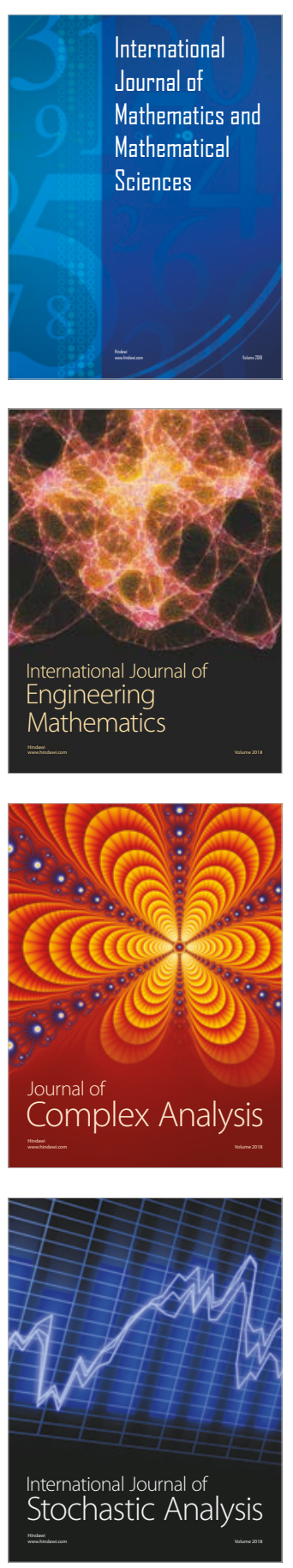
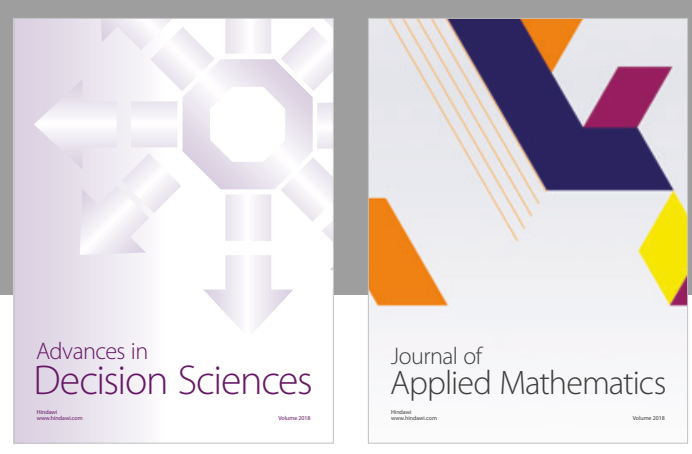

Journal of

Applied Mathematics
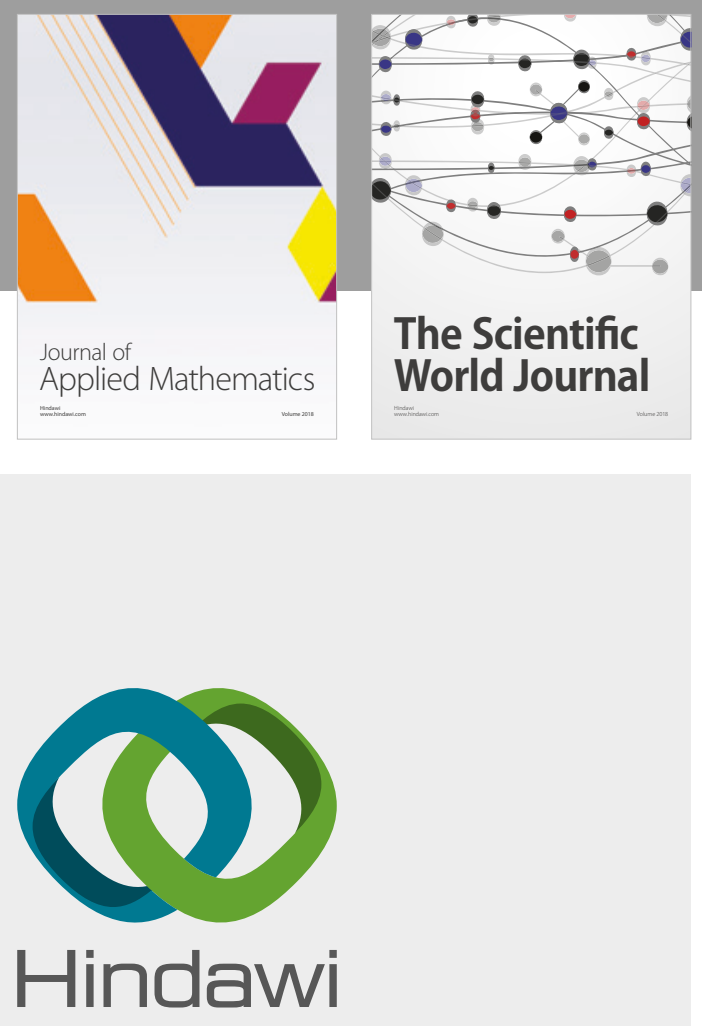

Submit your manuscripts at

www.hindawi.com

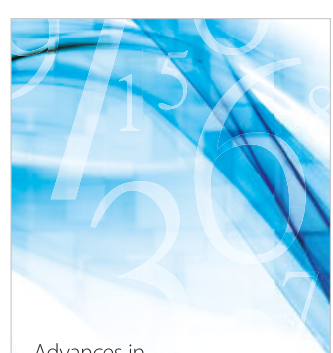

Advances in
Numerical Analysis
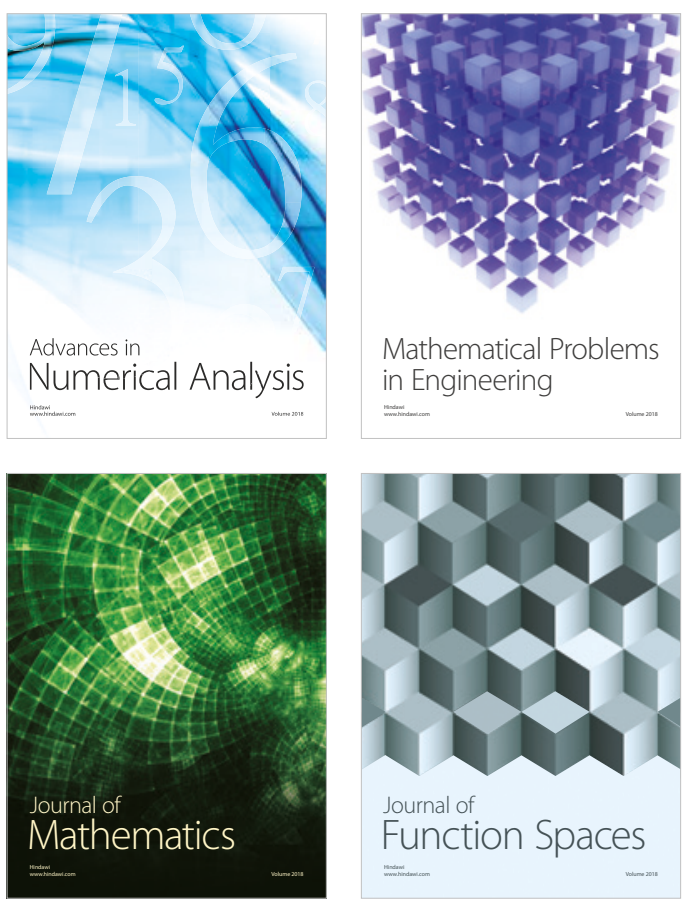

Mathematical Problems in Engineering

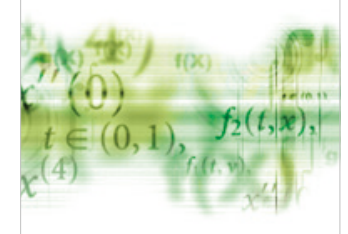

International Journal of

Differential Equations

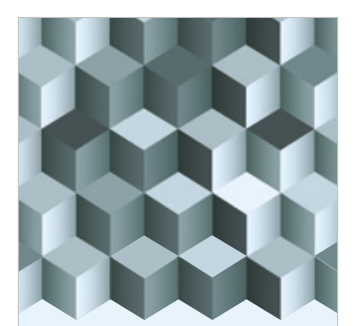

Journal of

Function Spaces

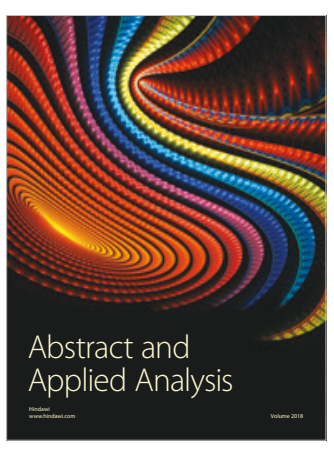

The Scientific

World Journal

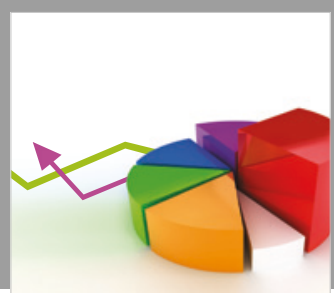

Journal of

Probability and Statistics
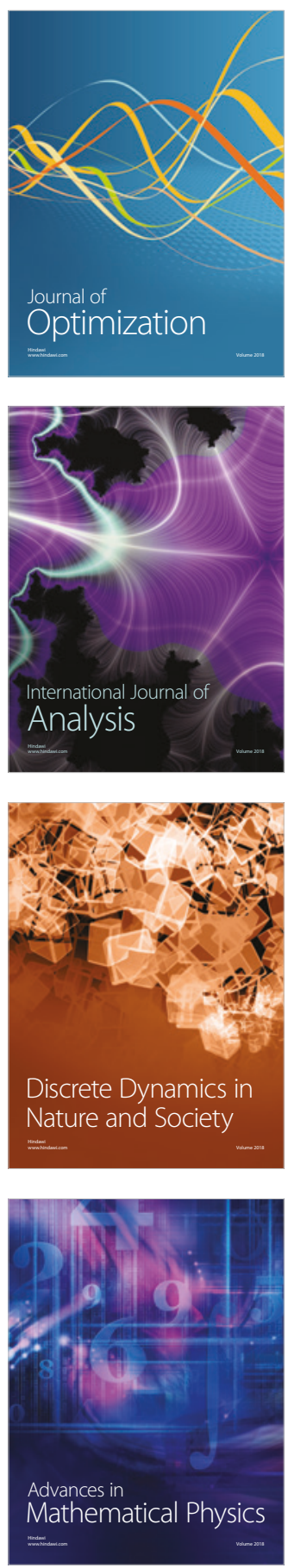